\title{
Hitting Probabilities and the Hausdorff Dimension of the Inverse Images of Anisotropic Gaussian Random Fields
}

\author{
Hermine Biermé \\ Université Paris Descartes \\ Céline Lacaux \\ Institut Élie Cartan, Nancy \\ Yimin Xiao \\ Michigan State University
}

August 22, 2008

\begin{abstract}
Let $X=\left\{X(t), t \in \mathbb{R}^{N}\right\}$ be a Gaussian random field with values in $\mathbb{R}^{d}$ defined by

$$
X(t)=\left(X_{1}(t), \ldots, X_{d}(t)\right),
$$

where $X_{1}, \ldots, X_{d}$ are independent copies of a centered Gaussian random field $X_{0}$. Under certain general conditions on $X_{0}$, we study the hitting probabilities of $X$ and determine the Hausdorff dimension of the inverse image $X^{-1}(F)$, where $F \subseteq \mathbb{R}^{d}$ is a non-random Borel set.

The class of Gaussian random fields that satisfy our conditions includes not only fractional Brownian motion, the Brownian sheet, but also such anisotropic fields as fractional Brownian sheets, solutions to stochastic heat equation driven by space-time white noise and the operator-scaling Gaussian random fields with stationary increments constructed in [4].
\end{abstract}

RunNing HeAD: Inverse Images of Anisotropic Gaussian Random Fields

2000 AMS Classification numbers: 60G60; 60G15; 60G17; 28A80.

KEY WORDS: Gaussian random fields; anisotropy; fractional Brownian sheet; stochastic heat equation; hitting probability; Hausdorff dimension; inverse image.

\section{Introduction}

In recent years, several classes of anisotropic Gaussian random fields have arisen naturally in the studies of random fields, stochastic partial differential equations as well as in many applied areas including image processing, hydrology, geostatistics and spatial statistics (see [23] and the references therein for more information).

Typical examples of anisotropic Gaussian random fields are fractional Brownian sheets and the solution to the stochastic heat equation. It has been known that the sample path properties such as fractal dimensions of these anisotropic Gaussian random fields can be very different from those of isotropic ones such as Lévy's fractional Brownian motion; see, for example, $[1,5,6,16,21]$. Recently, Xiao [23] studied systematically the analytic and geometric properties of anisotropic Gaussian random fields under certain general conditions, 
with the aim of characterizing their anisotropic nature by a multiparameter index $H=$ $\left(H_{1}, \ldots, H_{N}\right) \in(0,1)^{N}$. This index is often related to the operator-self-similarity or multiself-similarity of the Gaussian random field under study.

Let $X=\left\{X(t), t \in \mathbb{R}^{N}\right\}$ be a Gaussian random field with values in $\mathbb{R}^{d}$ defined on a probability space $(\Omega, \mathcal{F}, \mathbb{P})$ by

$$
X(t)=\left(X_{1}(t), \ldots, X_{d}(t)\right) .
$$

We will call $X$ an $(N, d)$-Gaussian random field. For any Borel set $F \subseteq \mathbb{R}^{d}$, its inverse image under $X$ is defined by

$$
X^{-1}(F)=\left\{t \in \mathbb{R}^{N}: X(t) \in F\right\} .
$$

In particular, if $F=\{x\}\left(x \in \mathbb{R}^{d}\right)$, then $X^{-1}(\{x\})$ is the level set of $X$.

In the studies of random fields, it is interesting to consider the following questions:

(i) Given a non-random Borel set $F \subseteq \mathbb{R}^{d}$, when is it non-polar for $X$ in the sense that $\mathbb{P}\left\{X^{-1}(F) \neq \varnothing\right\}>0$ ?

(ii) If $\mathbb{P}\left\{X^{-1}(F) \neq \varnothing\right\}>0$, what is the Hausdorff dimension of the inverse image $X^{-1}(F)$ ?

In this paper, we will use $\operatorname{dim}_{\mathrm{H}}$ to denote Hausdorff dimension, and refer to [8] and [11] for its definition and basic properties.

Question (i) is an important question in potential theory of random fields and complete answer has only been known for a few types of random fields with certain Markovian structures. Of particular significance is the result due to Khoshnevisan and Shi [14], who proved that if $W=\left\{W(t), t \in \mathbb{R}_{+}^{N}\right\}$ is the $(N, d)$-Brownian sheet, then for every Borel set $F \subseteq \mathbb{R}^{d}$,

$$
\mathbb{P}\left\{W^{-1}(F) \cap(0, \infty)^{N} \neq \varnothing\right\}>0 \Longleftrightarrow \mathcal{C}_{d-2 N}(F)>0 .
$$

Here and in the sequel, $\mathcal{C}_{\alpha}$ denotes the Bessel-Riesz capacity of order $\alpha$ defined by

$$
\mathcal{C}_{\alpha}(F)=\left[\inf _{\mu \in \mathcal{P}(F)} \int_{\mathbb{R}^{d}} \int_{\mathbb{R}^{d}} f_{\alpha}(\|x-y\|) \mu(d x) \mu(d y)\right]^{-1}
$$

where $\mathcal{P}(F)$ is the family of probability measures carried by $F$ and the function $f_{\alpha}:(0, \infty) \rightarrow$ $(0, \infty)$ is defined by

$$
f_{\alpha}(r)= \begin{cases}r^{-\alpha} & \text { if } \alpha>0, \\ \log \left(\frac{e}{r \wedge 1}\right) & \text { if } \alpha=0, \\ 1 & \text { if } \alpha<0 .\end{cases}
$$

Dalang and Nualart [7] extended the methods of Khoshnevisan and Shi [14] and proved similar results for the solution of a system of $d$ nonlinear hyperbolic stochastic partial differential equations with two variables.

For random fields with general dependence structures, it is more difficult to establish results of the type (1.2). Some sufficient conditions and necessary conditions for $\mathbb{P}\left\{X^{-1}(F) \neq\right.$ $\varnothing\}>0$ have been established by Testard [18] and Xiao [22] for fractional Brownian motion, by Dalang, Khoshnevisan and Nualart $[5,6]$ for the solutions to non-linear stochastic heat equations with additive and multiplicative noises, and by Xiao [23] for a large class of Gaussian random fields satisfying Conditions (C1) and (C2) (see Section 2 for the precise assumptions). Our Theorem 2.1 below unifies and, in some cases, strengthens the results in the aforementioned works. 
The main objective of this paper is to determine the Hausdorff dimension of the inverse image $X^{-1}(F)$ for Gaussian random fields satisfying Conditions (C1) and (C2). The analogous problem for Lévy processes can be solved by applying potential theory of Lévy processes and a subordination technique, see Hawkes [9] and Khoshnevisan and Xiao [13] for more details. However, when $X$ is a non-Markovian process or a Gaussian random field and $F \subseteq \mathbb{R}^{d}$ is a general Borel set, few results on the geometric properties of $X^{-1}(F)$ are available. Testard [18] obtained an upper bound for $\operatorname{dim}_{\mathrm{H}} B^{-1}(F)$ when $B=\left\{B(t), t \in \mathbb{R}^{N}\right\}$ is a fractional Brownian motion of index $\alpha \in(0,1)$, which is a centered Gaussian random field with values in $\mathbb{R}^{d}$ and covariance function given by

$$
\mathbb{E}\left[B_{i}(s) B_{j}(t)\right]=\frac{1}{2} \delta_{i j}\left(\|s\|^{2 \alpha}+\|t\|^{2 \alpha}-\|s-t\|^{2 \alpha}\right), \quad \forall s, t \in \mathbb{R}^{N},
$$

where $\|\cdot\|$ denotes the Euclidean norm in $\mathbb{R}^{N}$ and $\delta_{i j}=1$ if $i=j$ and 0 otherwise. Monrad and Pitt [17] proved that, if $N>\alpha d$, then almost surely

$$
\operatorname{dim}_{\mathrm{H}} B^{-1}(F)=N-\alpha d+\alpha \operatorname{dim}_{\mathrm{H}} F \quad \text { for all Borel sets } F \subseteq \mathbb{R}^{d} .
$$

Note that the exceptional null event on which (1.6) does not hold is independent of $F$, so such a result is called a uniform Hausdorff dimension result. The method of Monrad and Pitt [17] relies on rather strong conditions such as $N>\alpha d$ and the strong local nondeterminism of fractional Brownian motion. In Theorems 2.3 and 2.5 below, we determine $\operatorname{dim}_{\mathrm{H}} X^{-1}(F)$ for much more general Gaussian random fields. We should also point out that, compared with the isotropic case, the anisotropic nature of $X$ induces far richer fractal structure into $X^{-1}(F)$.

The rest of this paper is organized as follows. In Section 2, we provide the general assumptions on the Gaussian random fields under investigation and state the main results (i.e., Theorems 2.1, 2.3 and 2.5). Their proofs are given in Section 3. In Section 4 we apply our results to solutions of a system of $d$ nonlinear stochastic heat equations considered by Dalang, Khoshnevisan and Nualart [5] and determine the Hausdorff dimension of the inverse image $u^{-1}(F)$ of the solution $u=\{u(t, x), t \in[0, T], x \in[0,1]\}$, where $F \subseteq \mathbb{R}^{d}$ is an arbitrary non-random Borel set. This extends the results on the Hausdorff dimension of the level sets of $u$ in Dalang, Khoshnevisan and Nualart [5].

Throughout this paper we will use $c$ to denote an unspecified positive and finite constant which may not be the same in each occurrence. More specific constants in Section $i$ are numbered as $c_{i, 1}, c_{i, 2}, \ldots$

Acknowledgment The authors are grateful to F. Baudoin and A. Estrade since this work was partially supported by ANR grants ANR-05-BLAN-0017 and ANR-06-BLAN-0289. The research of Y. Xiao is also supported partially by NSF grant DMS-0706728.

This paper was finished while Y. Xiao was visiting the Statistical \& Applied Mathematical Sciences Institute (SAMSI). He thanks the staff of SAMSI for their support and the good working conditions.

The authors are deeply indebted to Kenneth Falconer for stimulating discussions, and to an anonymous referee for his/her corrections and suggestions which have lead to improvement of the manuscript. 


\section{Main results}

Let $\left(H_{1}, \ldots, H_{N}\right) \in(0,1)^{N}$ be a fixed vector and, for $a, b \in \mathbb{R}^{N}$ with $a_{j}<b_{j}(j=1, \ldots, N)$, let $I=[a, b]:=\prod_{j=1}^{N}\left[a_{j}, b_{j}\right] \subseteq \mathbb{R}^{N}$ denote an interval (or a rectangle). For simplicity, we set $I=\left[\varepsilon_{0}, 1\right]^{N}$, where $\varepsilon_{0} \in(0,1)$ is a fixed constant. For a given concrete Gaussian random field, the $\sigma$-stability of Hausdorff dimension (cf. [8]) will make it clear when one can replace $\left[\varepsilon_{0}, 1\right]^{N}$ by $[0,1]^{N}$ in Theorems 2.3 and 2.5 .

Let $X=\left\{X(t), t \in \mathbb{R}^{N}\right\}$ be an $(N, d)$-Gaussian random field defined by (1.1). Throughout this paper we assume that the coordinate processes $X_{1}, \ldots, X_{d}$ are independent copies of a real-valued, centered Gaussian random field $X_{0}=\left\{X_{0}(t), t \in \mathbb{R}^{N}\right\}$. We assume that $X_{0}$ satisfies the following general conditions:

(C1) There exist positive and finite constants $c_{2,1}, c_{2,2}$ and $c_{2,3}$ such that $\mathbb{E}\left[X_{0}(t)^{2}\right] \geq c_{2,1}$ for all $t \in I$ and

$$
c_{2,2} \sum_{j=1}^{N}\left|s_{j}-t_{j}\right|^{2 H_{j}} \leq \mathbb{E}\left[\left(X_{0}(s)-X_{0}(t)\right)^{2}\right] \leq c_{2,3} \sum_{j=1}^{N}\left|s_{j}-t_{j}\right|^{2 H_{j}}, \quad \forall s, t \in I .
$$

(C2) There exists a constant $c_{2,4}>0$ such that for all $s, t \in I$,

$$
\operatorname{Var}\left(X_{0}(t) \mid X_{0}(s)\right) \geq c_{2,4} \sum_{j=1}^{N}\left|s_{j}-t_{j}\right|^{2 H_{j}} .
$$

Here $\operatorname{Var}\left(X_{0}(t) \mid X_{0}(s)\right)$ denotes the conditional variance of $X_{0}(t)$ given $X_{0}(s)$.

It will be helpful to note that both (2.1) and (2.2) can be expressed in terms of the following metric $\rho$ on $\mathbb{R}^{N}$ :

$$
\rho(s, t)=\sum_{j=1}^{N}\left|s_{j}-t_{j}\right|^{H_{j}}, \quad \forall s, t \in \mathbb{R}^{N},
$$

since there exist positive constants $c_{2,5}$ and $c_{2,6}$ such that

$$
c_{2,5} \sum_{j=1}^{N}\left|s_{j}-t_{j}\right|^{2 H_{j}} \leq \rho(s, t)^{2} \leq c_{2,6} \sum_{j=1}^{N}\left|s_{j}-t_{j}\right|^{2 H_{j}}, \quad \forall s, t \in \mathbb{R}^{N} .
$$

The following are some remarks about Conditions $(\mathrm{C} 1)$ and $(\mathrm{C} 2)$.

- Condition " $\mathbb{E}\left[X_{0}(t)^{2}\right] \geq c_{2,1}$ for all $t \in I$ " in $(\mathrm{C} 1)$ assumes that $X$ is non-degenerate on $I$. This condition is needed to avoid some trivial cases in studying hitting probability of $X$ and $\operatorname{dim}_{\mathrm{H}}\left(X^{-1}(F) \cap I\right)$.

- Under Condition (C1), $X$ has a version which has continuous sample functions on $I$ almost surely. Henceforth we will assume without loss of generality that the Gaussian random field $X$ has continuous sample paths. 
- Conditions $(\mathrm{C} 1)$ and $(\mathrm{C} 2)$ are closely related. It is easy to see that $(\mathrm{C} 1)$ implies that $\operatorname{Var}\left(X_{0}(t) \mid X_{0}(s)\right) \leq c_{2,3} \sum_{j=1}^{N}\left|s_{j}-t_{j}\right|^{2 H_{j}}$ for all $s, t \in I$ and, on the other hand, (C2) implies $\sigma^{2}(s, t) \geq c_{2,4} \sum_{j=1}^{N}\left|s_{j}-t_{j}\right|^{2 H_{j}}$, where $\sigma^{2}(s, t)$ denotes $\mathbb{E}\left[\left(X_{0}(s)-X_{0}(t)\right)^{2}\right]$. Moreover, if the function $\sigma(0, t)$ satisfies certain smoothness condition, say, it has continuous first order partial derivatives on $I$, then one can show that $(\mathrm{C} 1)$ implies $(\mathrm{C} 2)$ by using the following fact (which can be verified easily): If $(U, V)$ is a centered Gaussian vector, then

$$
\operatorname{Var}(U \mid V)=\frac{\left(\rho_{U, V}^{2}-\left(\sigma_{U}-\sigma_{V}\right)^{2}\right)\left(\left(\sigma_{U}+\sigma_{V}\right)^{2}-\rho_{U, V}^{2}\right)}{4 \sigma_{V}^{2}},
$$

where $\rho_{U, V}^{2}=\mathbb{E}\left[(U-V)^{2}\right], \sigma_{U}^{2}=\mathbb{E}\left(U^{2}\right)$ and $\sigma_{V}^{2}=\mathbb{E}\left(V^{2}\right)$.

- Condition (C2) can be referred to as "two-point local nondeterminism" with index $H=\left(H_{1}, \ldots, H_{N}\right)$. See [23] for more information on properties of local nondeterminism and their applications.

We point out that the class of Gaussian random fields that satisfy Conditions (C1) and (C2) is large. It includes not only the well-known fractional Brownian motion and the Brownian sheet, but also such anisotropic random fields as fractional Brownian sheets ([1]), solutions to stochastic heat equation driven by space-time white noise ([5], [6], [16]) and many more. The results in this paper can be extended further so that they can be applied to the operator-scaling Gaussian fields with stationary increments constructed in [4]; see Remark 2.7 below.

Now we state the main results of this paper. We will always assume $F \subseteq \mathbb{R}^{d}$ to be non-empty and, except in Remark 2.8, non-random. Theorem 2.1 is concerned with the hitting probabilities of $X$ and provides a necessary condition and a sufficient condition for $\mathbb{P}\left\{X^{-1}(F) \cap I \neq \varnothing\right\}>0$.

Theorem 2.1 Let $X=\left\{X(t), t \in \mathbb{R}^{N}\right\}$ be an $(N, d)$-Gaussian random field defined by (1.1) and assume that $X_{0}$ satisfies Conditions (C1) and (C2). If $F \subseteq \mathbb{R}^{d}$ is a Borel set, then

$$
c_{2,7} \mathcal{C}_{d-Q}(F) \leq \mathbb{P}\left\{X^{-1}(F) \cap I \neq \varnothing\right\} \leq c_{2,8} \mathcal{H}_{d-Q}(F),
$$

where $c_{2,7}$ and $c_{2,8}$ are positive constants depending on $I, F$ and $H$ only. In the above, $Q:=\sum_{j=1}^{N} 1 / H_{j}$ and $\mathcal{H}_{q}(F)$ is defined as the q-dimensional Hausdorff measure of $F$ when $q>0$, and $\mathcal{H}_{q}(F)=1$ whenever $q \leq 0$.

\section{Remark 2.2}

- Note that if $0 \in F$, then the second inequality in (2.5) might not hold without the condition " $\mathbb{E}\left[X_{0}(t)^{2}\right] \geq c_{2,1}$ for all $t \in I$ " in $(\mathrm{C} 1)$.

- Let $F \subseteq \mathbb{R}^{d}$ be a Borel set and $Q=\sum_{j=1}^{N} 1 / H_{j}$. Theorem 2.1 implies the following statements:

(i) If $\operatorname{dim}_{\mathrm{H}} F<d-Q$, then $X^{-1}(F) \cap I=\varnothing$ a.s.

(ii) If $\operatorname{dim}_{\mathrm{H}} F>d-Q$, then $\mathbb{P}\left\{X^{-1}(F) \cap I \neq \varnothing\right\}>0$. 
- A natural conjecture is that, at least for a large subclass of Gaussian random fields satisfying the conditions of Theorem $2.1, \mathbb{P}\left\{X^{-1}(F) \cap I \neq \varnothing\right\}>0 \Longleftrightarrow \mathcal{C}_{d-Q}(F)>0$.

Next we consider the Hausdorff dimension of the inverse image $X^{-1}(F) \cap I$. In the special case of fractional Brownian motion, this problem was considered by Testard [18] and Monrad and Pitt [17]. The following two theorems determine $\operatorname{dim}_{\mathrm{H}}\left(X^{-1}(F) \cap I\right)$ in terms of $\operatorname{dim}_{\mathrm{H}} F$ and the parameters $N, d$ and $H \in(0,1)^{N}$ of $X$. Compared with the results for fractional Brownian motion, we see that the fractal structure of the inverse images of an anisotropic random field is much richer.

For convenience we will always assume

$$
0<H_{1} \leq H_{2} \leq \cdots \leq H_{N}<1 .
$$

Theorem 2.3 Let $X=\left\{X(t), t \in \mathbb{R}^{N}\right\}$ be an $(N, d)$-Gaussian random field defined by (1.1) with $X_{0}$ satisfying Conditions (C1) and (C2). Let $F \subseteq \mathbb{R}^{d}$ be a Borel set such that $\operatorname{dim}_{\mathrm{H}} F \geq$ $d-Q$. Then the following statements hold:

(i) Almost surely

$$
\operatorname{dim}_{\mathrm{H}}\left(X^{-1}(F) \cap I\right) \leq \min _{1 \leq k \leq N}\left\{\sum_{j=1}^{k} \frac{H_{k}}{H_{j}}+N-k-H_{k}\left(d-\operatorname{dim}_{\mathrm{H}} F\right)\right\} .
$$

In particular, if $\operatorname{dim}_{\mathrm{H}} F=d-Q$, then $\operatorname{dim}_{\mathrm{H}}\left(X^{-1}(F) \cap I\right)=0$ a.s.

(ii) If $\operatorname{dim}_{\mathrm{H}} F>d-Q$, then for every $\varepsilon>0$,

$$
\operatorname{dim}_{\mathrm{H}}\left(X^{-1}(F) \cap I\right) \geq \min _{1 \leq k \leq N}\left\{\sum_{j=1}^{k} \frac{H_{k}}{H_{j}}+N-k-H_{k}\left(d-\operatorname{dim}_{\mathrm{H}} F\right)\right\}-\varepsilon
$$

on an event of positive probability (which may depend on $\varepsilon$ ). In the special case when $\operatorname{dim}_{\mathrm{H}} F=$ 0 and $d<Q$, we have

$$
\operatorname{dim}_{\mathrm{H}}\left(X^{-1}(F) \cap I\right)=\min _{1 \leq k \leq N}\left\{\sum_{j=1}^{k} \frac{H_{k}}{H_{j}}+N-k-H_{k} d\right\}
$$

on an event of positive probability.

As in [9] and [13], we can combine (2.7) and (2.8) in Theorem 2.3 and write

$$
\left\|\operatorname{dim}_{\mathrm{H}}\left(X^{-1}(F) \cap I\right)\right\|_{L^{\infty}(\mathbb{P})}=\min _{1 \leq k \leq N}\left\{\sum_{j=1}^{k} \frac{H_{k}}{H_{j}}+N-k-H_{k}\left(d-\operatorname{dim}_{\mathrm{H}} F\right)\right\} .
$$

Here and in the sequel, for any function $Y: \Omega \rightarrow \mathbb{R}_{+},\|Y\|_{L^{\infty}(\mathbb{P})}$ is defined as

$$
\|Y\|_{L^{\infty}(\mathbb{P})}=\sup \{\theta: Y \geq \theta \text { on an event } E \text { with } \mathbb{P}(E)>0\} .
$$

Note that (2.10) gives essential bounds for the Hausdorff dimension of the inverse image, it is more desirable to establish an exact formula for $\operatorname{dim}_{\mathrm{H}}\left(X^{-1}(F) \cap I\right)$. This amounts to strengthen the conclusion in Part (ii) of Theorem 2.3 so that the positive probability in (2.8) is independent of $\varepsilon$. For this purpose, we introduce the following condition (S), which contains more information on the geometry of $F$ than its Hausdorff dimension alone. 
(S): There is a finite constant $c_{2,9} \geq 1$ such that, for every $\gamma \in\left(0, \operatorname{dim}_{\mathrm{H}} F\right)$, there exists a probability measure $\mu_{0, \gamma}$ (which may depend on $\gamma$ ) with compact support in $F$ such that

$$
\mu_{0, \gamma}(B(x, r)) \leq c_{2,9} r^{\gamma} \quad \text { for all } \quad x \in \mathbb{R}^{d} \text { and } r>0 \text {. }
$$

Remark 2.4 The key feature of Condition $(S)$ is that the constant $c_{2,9}$ is independent of $\gamma<\operatorname{dim}_{\mathrm{H}} F$, even though the probability measure $\mu_{0, \gamma}$ may depend on $\gamma$. This assumption is stronger than the conclusion of Frostman's lemma (where both $c_{2,9}$ and $\mu_{0, \gamma}$ may depend on $\gamma$ ), and is verified by a large class of Borel sets including all self-similar sets satisfying the open set condition ([8]) and, more generally, the $d$-sets ([10]). If $F$ is a compact set in $\mathbb{R}^{d}$, then Condition $(S)$ is equivalent to the existence of a constant $c_{2,9} \geq 1$ and a probability measure $\mu$ on $F$ such that

$$
\mu(B(x, r)) \leq c_{2,9} r^{\operatorname{dim}_{\mathrm{H}} F} \quad \text { for all } x \in \mathbb{R}^{d} \text { and } r>0 .^{1}
$$

In fact, one direction is obvious. For the other direction, take $\mu$ to be an arbitrary subsequential weak limit of $\left\{\mu_{0, \gamma}\right\}$.

By examining the proof of Frostman's lemma in [11, p. 130-131], one sees that Condition $(S)$ is satisfied provided that the Borel set $F$ has the following property: There is a constant $c_{2,10}>0$ such that, for every $\gamma \in\left(0, \operatorname{dim}_{\mathrm{H}} F\right)$, one has

$$
\inf \left\{\sum_{n=1}^{\infty}\left(\operatorname{diam} B_{n}\right)^{\gamma}: F \subseteq \bigcup_{n=1}^{\infty} B_{n}\right\} \geq c_{2,10},
$$

where the infimum is taken over all coverings of $F$ by balls $\left\{B_{n}, n \geq 1\right\}$. However, we have not been able to fully characterize the family of Borel sets that satisfies Condition $(S)$.

The following theorem shows that Condition (S) is a sufficient (but not necessary) condition for the probability in (2.8) to be independent of $\varepsilon$. Because of (2.7) and (2.9), it suffices to consider Borel sets $F \subseteq \mathbb{R}^{d}$ that satisfy $\operatorname{dim}_{\mathrm{H}} F>\max \{d-Q, 0\}$.

Theorem 2.5 Let $X=\left\{X(t), t \in \mathbb{R}^{N}\right\}$ be as in Theorem 2.3 and let $F \subseteq \mathbb{R}^{d}$ be a bounded Borel set such that $\operatorname{dim}_{\mathrm{H}} F>\max \{d-Q, 0\}$ and satisfies the condition $(S)$. Then with positive probability,

$$
\begin{aligned}
& \operatorname{dim}_{\mathrm{H}}\left(X^{-1}(F) \cap I\right)=\min _{1 \leq k \leq N}\left\{\sum_{j=1}^{k} \frac{H_{k}}{H_{j}}+N-k-H_{k}\left(d-\operatorname{dim}_{\mathrm{H}} F\right)\right\} \\
& =\sum_{j=1}^{k} \frac{H_{k}}{H_{j}}+N-k-H_{k}\left(d-\operatorname{dim}_{\mathrm{H}} F\right), \quad \text { if } \sum_{j=1}^{k-1} \frac{1}{H_{j}} \leq d-\operatorname{dim}_{\mathrm{H}} F<\sum_{j=1}^{k} \frac{1}{H_{j}} .
\end{aligned}
$$

In the above we use the convention $\sum_{j=1}^{0} 1 / H_{j}=0$.

We end this section with the following remarks about Theorems 2.3 and 2.5.

\footnotetext{
${ }^{1}$ This statement and its proof were showed to us by the referee.
} 
Remark 2.6 In the special case of $F=\{x\}, X^{-1}(F) \cap I$ becomes the level set of $X$ on $I$ and the above Hausdorff dimension formula (2.9) was proved by Ayache and Xiao [1] for fractional Brownian sheets, by Wu and Xiao [20] for the solutions of stochastic heat equation driven by a space-time white noise, and by Xiao [23] for Gaussian random fields satisfying Conditions (C1) and (C2). Theorems 2.3 and 2.5 extend their results and, as we will see in the next section, their proofs rely on some new ingredients.

Remark 2.7 From the proof of Theorem 2.3 given in Section 3 one can see that its conclusions still hold if (2.1) and (2.2) in Conditions (C1) and (C2) are replaced respectively by the following weaker conditions: For all $\varepsilon>0$, there exist constants $c_{2, i}^{\prime}(i=2,3,4)$ which may depend on $\varepsilon$ such that,

$$
c_{2,2}^{\prime} \sum_{j=1}^{N}\left|s_{j}-t_{j}\right|^{2 H_{j}+\varepsilon} \leq \mathbb{E}\left[\left(X_{0}(s)-X_{0}(t)\right)^{2}\right] \leq c_{2,3}^{\prime} \sum_{j=1}^{N}\left|s_{j}-t_{j}\right|^{2 H_{j}-\varepsilon}, \quad \forall s, t \in I ;
$$

and

$$
\operatorname{Var}\left(X_{0}(t) \mid X_{0}(s)\right) \geq c_{2,4}^{\prime} \sum_{j=1}^{N}\left|s_{j}-t_{j}\right|^{2 H_{j}+\varepsilon}, \quad \forall s, t \in I .
$$

It can be proven that the operator-scaling Gaussian fields constructed in [4] satisfy conditions (2.15) and (2.16), while Conditions (C1) and (C2) are only satisfied by random fields with diagonal scaling matrices. We refer to [2] and [3] for further information.

Remark 2.8 Note that the event described by (2.14) depends on $F$. In light of the result (1.6) of Monrad and Pitt [17], we may ask the following natural question: When $\sum_{j=1}^{N} 1 / H_{j}>d$, is it possible to have a single event $\Omega_{1} \subseteq \Omega$ of positive probability such that on $\Omega_{1}(2.14)$ holds for all Borel sets $F \subseteq \mathbb{R}^{d}$ ? This is simply referred to as a uniform Hausdorff dimension problem for the inverse images of $X$. An affirmative answer will make the formula (2.14) applicable to random Borel sets $F$ as well.

If, in addition to Conditions $(\mathrm{C} 1)$ and $(\mathrm{C} 2)$, we also assume that Condition (C3) or $\left(\mathrm{C} 3^{\prime}\right)$ in [23] holds and $H_{1}=H_{2}=\cdots=H_{N}$, then one can modify the proofs in [17] to prove that the answer to the above question is affirmative when $N>H_{1} d$. In general, it can be proved that the upper bound (2.7) holds almost surely for all Borel sets $F \subseteq \mathbb{R}^{d}$. However the lower bound might not hold uniformly due to the anisotropy of $X$.

Remark 2.9 Another useful dimension for studying fractals is packing dimension, denoted by $\operatorname{dim}_{\mathrm{P}}$, see [8] for its definition and properties. We mention that the problem of finding the packing dimension of $X^{-1}(F)$ remains widely open. Even though one can apply Lemma 3.1 and a covering argument to derive that under the conditions of Theorem 2.3,

$$
\operatorname{dim}_{\mathrm{P}}\left(X^{-1}(F) \cap I\right) \leq \min _{1 \leq k \leq N}\left\{\sum_{j=1}^{k} \frac{H_{k}}{H_{j}}+N-k-H_{k}\left(d-\operatorname{dim}_{\mathrm{P}} F\right)\right\}, \quad \text { a.s. }
$$

it is possible that, for a general Borel set $F \subseteq \mathbb{R}^{d}$, the inequality in (2.17) might be strict. New ideas may be needed for solving this problem. 


\section{Proofs}

In this section we prove the theorems stated in Section 2. The proofs of Theorem 2.1 and Theorem 2.3 are divided into proving the upper and lower bounds for $\mathbb{P}\left\{X^{-1}(F) \cap I \neq \varnothing\right\}$ and $\operatorname{dim}_{\mathrm{H}}\left(X^{-1}(F) \cap I\right)$ separately. The upper bounds will be proved by using a covering argument, and the lower bounds will be proved by constructing a random measure on $X^{-1}(F) \cap I$. These methods are similar to those in [11], [18] and [22] for studying the level sets and hitting probabilities of fractional Brownian motion. However, in order to deal with anisotropic Gaussian random fields in general, we have to rely also on some new ingredients such as Lemma 3.1 and Lemma 3.2 below.

We will apply the following lemma on the hitting probability of $X$ to prove the upper bounds in (2.5) and (2.7). A slightly stronger version of this lemma was provided in [23] with a longer proof. For the sake of completeness, we will provide a simpler proof by modifying the argument in the proof of Proposition 4.4 in Dalang, Khoshnevisan and Nualart [5].

Lemma 3.1 Let $X=\left\{X(t), t \in \mathbb{R}^{N}\right\}$ be an $(N, d)$-Gaussian random field defined by (1.1) with $X_{0}$ satisfying Conditions (C1) and (C2). For any constant $M>0$, there exist positive constants $c_{3,1}$ and $\delta_{0}$ such that for all $r \in\left(0, \delta_{0}\right), t \in I$ and all $x \in[-M, M]^{d}$,

$$
\mathbb{P}\left\{\inf _{s \in B_{\rho}(t, r) \cap I}\|X(s)-x\| \leq r\right\} \leq c_{3,1} r^{d} .
$$

In the above $B_{\rho}(t, r)=\left\{s \in \mathbb{R}^{N}: \rho(s, t) \leq r\right\}$ denotes the closed ball of radius $r$ in the metric $\rho$ in $\mathbb{R}^{N}$ defined by (2.3).

Proof Since the coordinate processes of $X$ are independent copies of $X_{0}$, it is sufficient to prove (3.1) for $X_{0}$. Note that for any $s \in I$, we have

$$
\mathbb{E}\left(X_{0}(s) \mid X_{0}(t)\right)=\frac{\mathbb{E}\left(X_{0}(s) X_{0}(t)\right)}{\mathbb{E}\left(X_{0}(t)^{2}\right)} X_{0}(t):=c(s, t) X_{0}(t)
$$

Then the Gaussian random variables $X_{0}(s)-c(s, t) X_{0}(t)(s \in I)$ and $X_{0}(t)$ are independent. Let

$$
Z(t, r)=\sup _{s \in B_{\rho}(t, r) \cap I}\left|X_{0}(s)-c(s, t) X_{0}(t)\right| .
$$

Then $Z(t, r)$ is independent of $X_{0}(t)$. It follows from triangle's inequality that

$$
\begin{aligned}
& \mathbb{P}\left\{\inf _{s \in B_{\rho}(t, r) \cap I}\left|X_{0}(s)-x\right| \leq r\right\} \\
& \leq \mathbb{P}\left\{\inf _{s \in B_{\rho}(t, r) \cap I}\left|c(s, t)\left(X_{0}(t)-x\right)\right| \leq r+Z(t, r)+\sup _{s \in B_{\rho}(t, r) \cap I}|(1-c(s, t)) x|\right\} .
\end{aligned}
$$

The Cauchy-Schwarz inequality and Condition (C1) imply that for all $s, t \in I$,

$$
|1-c(s, t)|=\frac{\left|\mathbb{E}\left[X_{0}(t)\left(X_{0}(t)-X_{0}(s)\right)\right]\right|}{\mathbb{E}\left(X_{0}(t)^{2}\right)} \leq\left(\frac{c_{2,3}}{c_{2,1}} \sum_{j=1}^{N}\left|s_{j}-t_{j}\right|^{2 H_{j}}\right)^{1 / 2} .
$$


Hence there exists a constant $\delta_{0}>0$ such that for all $r \in\left(0, \delta_{0}\right)$ and $s \in B_{\rho}(t, r) \cap I$, we have $1 / 2 \leq c(s, t) \leq 1$. Moreover, for all $r \in\left(0, \delta_{0}\right)$, all $s \in B_{\rho}(t, r) \cap I$ and all $x \in[-M, M]$,

$$
|(1-c(s, t)) x| \leq \frac{M r}{2} \text {. }
$$

Combining the above with (3.3) it follows that for all $x \in[-M, M]$ and $r \in\left(0, \delta_{0}\right)$,

$\mathbb{P}\left\{\inf _{s \in B_{\rho}(t, r) \cap I}\left|X_{0}(s)-x\right| \leq r\right\} \leq \mathbb{P}\left\{x-(M+2) r-2 Z(t, r) \leq X_{0}(t) \leq x+(M+2) r+2 Z(t, r)\right\}$.

Then using the independence of $X_{0}(t)$ and $Z(t, r)$ and Condition $(\mathrm{C} 1)$ we obtain that for all $x \in[-M, M]$ and $r \in\left(0, \delta_{0}\right)$,

$$
\mathbb{P}\left\{\inf _{s \in B_{\rho}(t, r) \cap I}\left|X_{0}(s)-x\right| \leq r\right\} \leq c_{3,2} \mathbb{E}(r+Z(t, r))=c_{3,2} r+c_{3,2} \mathbb{E}(Z(t, r)),
$$

where $c_{3,2}$ is a finite positive constant which depends on $M$.

The last term in (3.5) can be estimated by applying Dudley's entropy theorem (see, e.g., [11], p. 219) to the Gaussian random field $Y(s)=X_{0}(s)-c(s, t) X_{0}(t)\left(s \in B_{\rho}(t, r) \cap I\right)$. To this end, note that $Y(t)=0$ and the canonical metric

$$
d\left(s, s^{\prime}\right):=\left\{\mathbb{E}\left(Y(s)-Y\left(s^{\prime}\right)\right)^{2}\right\}^{1 / 2} \leq c \rho\left(s, s^{\prime}\right)
$$

for all $s, s^{\prime} \in B_{\rho}(t, r) \cap I$. Denote by $D:=\sup _{s, s^{\prime} \in B_{\rho}(t, r) \cap I} d\left(s, s^{\prime}\right)$ and $N_{d}\left(B_{\rho}(t, r), \varepsilon\right)$ the $d$-diameter and the metric entropy number of $B_{\rho}(t, r)$, respectively. Then $D \leq c r$ and

$$
N_{d}\left(B_{\rho}(t, r), \varepsilon\right) \leq c\left(\frac{r}{\varepsilon}\right)^{Q} .
$$

It follows from Dudley's theorem that

$$
\mathbb{E}(Z(t, r)) \leq c \int_{0}^{D} \sqrt{\log N_{d}\left(B_{\rho}(t, r), \varepsilon\right)} d \varepsilon \leq c_{3,3} r .
$$

This and (3.5) together imply (3.1) for $d=1$. The proof of Lemma 3.1 is finished.

For proving the lower bounds in Theorems 2.1, 2.3 and Theorem 2.5 we will make use of the following lemma which extends a result of Testard [18] for fractional Brownian motion.

Lemma 3.2 Let $X=\left\{X(t), t \in \mathbb{R}^{N}\right\}$ be an $(N, d)$-Gaussian random field defined by (1.1) with $X_{0}$ satisfying Conditions (C1) and (C2). Then there exists a positive and finite constant $c_{3,4}$ such that for all $\varepsilon \in(0,1), s, t \in I$ and $x, y \in \mathbb{R}^{d}$, we have

$$
\begin{aligned}
& \int_{\mathbb{R}^{2 d}} e^{-i(\langle\xi, x\rangle+\langle\eta, y\rangle)} \exp \left(-\frac{1}{2}(\xi, \eta)\left(\varepsilon I_{2 d}+\operatorname{Cov}(X(s), X(t))\right)(\xi, \eta)^{\prime}\right) d \xi d \eta \\
& \quad \leq \frac{c_{3,4}}{\max \left\{\rho^{d}(s, t),\|x-y\|^{d}\right\}} .
\end{aligned}
$$

In the above, $I_{2 d}$ denotes the identity matrix of order $2 d, \operatorname{Cov}(X(s), X(t))$ denotes the covariance matrix of the random vector $(X(s), X(t))$, and $(\xi, \eta)^{\prime}$ is the transpose of the row vector $(\xi, \eta)$. 
Proof Since the coordinate processes $X_{1}, \ldots, X_{d}$ of $X$ are independent copies of $X_{0}$, we first consider the case when $d=1$.

For any $\varepsilon \in(0,1)$, let

$$
\begin{aligned}
\Phi_{\varepsilon}^{s, t}\left(\xi_{1}, \eta_{1}\right) & =\varepsilon\left(\xi_{1}^{2}+\eta_{1}^{2}\right)+\mathbb{E}\left(\xi_{1} X_{1}(s)+\eta_{1} X_{1}(t)\right)^{2} \\
& =\left(\varepsilon+\mathbb{E}\left(X_{1}(s)^{2}\right)\right) \xi_{1}^{2}+2 \xi_{1} \eta_{1} \mathbb{E}\left(X_{1}(s) X_{1}(t)\right)+\left(\varepsilon+\mathbb{E}\left(X_{1}(t)^{2}\right)\right) \eta_{1}^{2} .
\end{aligned}
$$

Denote by $\Gamma_{\varepsilon}(s, t)$ the symmetric matrix corresponding to the quadratic form (3.8). Then $\Gamma_{\varepsilon}(s, t)=\varepsilon I_{2}+\operatorname{Cov}\left(X_{1}(s), X_{1}(t)\right)$ and its inverse is given by

$$
\Gamma_{\varepsilon}^{-1}(s, t)=\frac{1}{\operatorname{det}\left(\Gamma_{\varepsilon}(s, t)\right)}\left(\begin{array}{cc}
\varepsilon+\mathbb{E}\left(X_{1}(t)^{2}\right) & -\mathbb{E}\left(X_{1}(s) X_{1}(t)\right) \\
-\mathbb{E}\left(X_{1}(s) X_{1}(t)\right) & \varepsilon+\mathbb{E}\left(X_{1}(s)^{2}\right)
\end{array}\right),
$$

where $\operatorname{det}\left(\Gamma_{\varepsilon}(s, t)\right)$ denotes the determinant of $\Gamma_{\varepsilon}(s, t)$. Since the symmetric matrix $\Gamma_{\varepsilon}(s, t)$ is positive definite for all $\varepsilon>0$, we have

$$
\begin{aligned}
\int_{\mathbb{R}^{2}} e^{-i\left(\xi_{1} x_{1}+\eta_{1} y_{1}\right)} \exp \left(-\frac{1}{2} \Phi_{\varepsilon}^{s, t}\left(\xi_{1}, \eta_{1}\right)\right) d \xi_{1} d \eta_{1} \\
=\frac{2 \pi}{\sqrt{\operatorname{det}\left(\Gamma_{\varepsilon}(s, t)\right)}} \exp \left(-\frac{1}{2}\left(x_{1}, y_{1}\right) \Gamma_{\varepsilon}^{-1}(s, t)\left(x_{1}, y_{1}\right)^{\prime}\right) .
\end{aligned}
$$

Let us remark that

$$
\begin{aligned}
\left(x_{1}, y_{1}\right) \Gamma_{\varepsilon}^{-1}(s, t)\left(x_{1}, y_{1}\right)^{\prime} & =\frac{1}{\operatorname{det}\left(\Gamma_{\varepsilon}(s, t)\right)}\left(\varepsilon\left(x_{1}^{2}+y_{1}^{2}\right)+\mathbb{E}\left[\left(x_{1} X_{1}(t)-y_{1} X_{1}(s)\right)^{2}\right]\right) \\
& \geq \frac{1}{\operatorname{det}\left(\Gamma_{\varepsilon}(s, t)\right)} \mathbb{E}\left[\left(x_{1} X_{1}(t)-y_{1} X_{1}(s)\right)^{2}\right] .
\end{aligned}
$$

We claim that for all $s, t \in I$ and all $x_{1}, y_{1} \in \mathbb{R}$,

$$
\mathbb{E}\left[\left(x_{1} X_{1}(t)-y_{1} X_{1}(s)\right)^{2}\right] \geq c_{3,5}\left(x_{1}-y_{1}\right)^{2},
$$

where $c_{3,5}>0$ is a constant depending only on $c_{2,1}, \ldots, c_{2,4}$ in Conditions $(\mathrm{C} 1)$ and $(\mathrm{C} 2)$.

In order to verify (3.12), we note that it is equivalent to prove the following: There exists a constant $c>0$ such that, for all $s, t \in I$, the quadratic form

$$
\mathbb{E}\left[\left(x_{1} X_{1}(s)-y_{1} X_{1}(t)\right)^{2}\right]-c\left(x_{1}-y_{1}\right)^{2}
$$

is positive semi-definite. Hence it is sufficient to show the existence of a constant $c>0$ such that

(i) $\mathbb{E}\left(X_{1}(s)^{2}\right) \geq c$ for all $s \in I$.

(ii) for all $s, t \in I$ we have

$$
\left(\mathbb{E}\left(X_{1}(s)^{2}\right)-c\right)\left(\mathbb{E}\left(X_{1}(t)^{2}\right)-c\right)-\left(\mathbb{E}\left(X_{1}(s) X_{1}(t)\right)-c\right)^{2} \geq 0
$$


Since Condition (C1) implies (i) holds for any $c \in\left(0, c_{2,1}\right]$, it remains to verify (ii) for $c>0$ small enough.

It can be seen that (3.14) holds for some $c>0$ if and only if

$$
c \leq \frac{\mathbb{E}\left(X_{1}(s)^{2}\right) \mathbb{E}\left(X_{1}(t)^{2}\right)-\left(\mathbb{E}\left(X_{1}(s) X_{1}(t)\right)\right)^{2}}{\mathbb{E}\left[\left(X_{1}(s)-X_{1}(t)\right)^{2}\right]}
$$

for all $s, t \in I$ with $s \neq t$. Observe that the numerator in (3.15) is $\operatorname{det} \operatorname{Cov}\left(X_{1}(s), X_{1}(t)\right)$. It follows from $(\mathrm{C} 1),(\mathrm{C} 2)$ and the identity that for any Gaussian vector $\left(Z_{1}, Z_{2}\right)$,

$$
\operatorname{det} \operatorname{Cov}\left(Z_{1}, Z_{2}\right)=\operatorname{Var}\left(Z_{1}\right) \operatorname{Var}\left(Z_{2} \mid Z_{1}\right)
$$

that

$$
\operatorname{det} \operatorname{Cov}\left(X_{1}(s), X_{1}(t)\right) \geq c_{2,1} c_{2,4} \sum_{j=1}^{N}\left|s_{j}-t_{j}\right|^{2 H_{j}} .
$$

This and (2.1) imply that the right-hand side of (3.15) is bounded from below by $c_{2,1} c_{2,4} / c_{2,3}$. By taking $0<c \leq \min \left\{c_{2,1}, c_{2,1} c_{2,4} / c_{2,3}\right\}$, we have verified (3.12).

Combining (3.10), (3.11) and (3.12) together, we obtain

$$
\begin{gathered}
\int_{\mathbb{R}^{2}} e^{-i\left(\xi_{1} x_{1}+\eta_{1} y_{1}\right)} \exp \left(-\frac{1}{2} \Phi_{\varepsilon}^{s, t}\left(\xi_{1}, \eta_{1}\right)\right) d \xi_{1} d \eta_{1} \\
\leq \frac{2 \pi}{\sqrt{\operatorname{det}\left(\Gamma_{\varepsilon}(s, t)\right)}} \exp \left(-\frac{c_{3,5}}{2} \frac{\left(x_{1}-y_{1}\right)^{2}}{\operatorname{det}\left(\Gamma_{\varepsilon}(s, t)\right)}\right) .
\end{gathered}
$$

Going back to $\mathbb{R}^{d}$, we derive from (3.18) that for all $\varepsilon>0$,

$$
\begin{gathered}
\int_{\mathbb{R}^{2 d}} e^{-i(\langle\xi, x\rangle+\langle\eta, y\rangle)} \exp \left(-\frac{1}{2}(\xi, \eta)\left(\varepsilon I_{2 d}+\operatorname{Cov}(X(s), X(t))\right)(\xi, \eta)^{\prime}\right) d \xi d \eta \\
\leq \frac{(2 \pi)^{d}}{\left[\operatorname{det}\left(\Gamma_{\varepsilon}(s, t)\right)\right]^{d / 2}} \exp \left(-\frac{c_{3,5}}{2} \frac{\|x-y\|^{2}}{\operatorname{det}\left(\Gamma_{\varepsilon}(s, t)\right)}\right) .
\end{gathered}
$$

Note that, if $\operatorname{det}\left(\Gamma_{\varepsilon}(s, t)\right) \geq\|x-y\|^{2}$, then

$$
\frac{(2 \pi)^{d}}{\left[\operatorname{det}\left(\Gamma_{\varepsilon}(s, t)\right)\right]^{d / 2}} \exp \left(-\frac{c_{3,5}}{2} \frac{\|x-y\|^{2}}{\operatorname{det}\left(\Gamma_{\varepsilon}(s, t)\right)}\right) \leq \frac{(2 \pi)^{d}}{\left[\operatorname{det}\left(\Gamma_{\varepsilon}(s, t)\right)\right]^{d / 2}} .
$$

On the other hand, if $\operatorname{det}\left(\Gamma_{\varepsilon}(s, t)\right)<\|x-y\|^{2}$, then we use the elementary inequality $x^{d / 2} e^{-c x} \leq c_{3,6}(\forall x>0)$ to obtain

$$
\frac{(2 \pi)^{d}}{\left[\operatorname{det}\left(\Gamma_{\varepsilon}(s, t)\right)\right]^{d / 2}} \exp \left(-\frac{c_{3,5}}{2} \frac{\|x-y\|^{2}}{\operatorname{det}\left(\Gamma_{\varepsilon}(s, t)\right)}\right) \leq \frac{c_{3,7}}{\|x-y\|^{d}}
$$

By (3.20) and (3.21) we obtain

$$
\frac{(2 \pi)^{d}}{\left[\operatorname{det}\left(\Gamma_{\varepsilon}(s, t)\right)\right]^{d / 2}} \exp \left(-\frac{c_{3,5}}{2} \frac{\|x-y\|^{2}}{\operatorname{det}\left(\Gamma_{\varepsilon}(s, t)\right)}\right) \leq \frac{c_{3,8}}{\max \left\{\left[\operatorname{det}\left(\Gamma_{\varepsilon}(s, t)\right)\right]^{d / 2},\|x-y\|^{d}\right\}} \text {. }
$$

Finally, we note that for all $s, t \in I$,

$$
\operatorname{det}\left(\Gamma_{\varepsilon}(s, t)\right) \geq \operatorname{det} \operatorname{Cov}\left(X_{1}(s), X_{1}(t)\right) \geq c_{2,1} c_{2,4} \rho(s, t)^{2} .
$$

Therefore (3.7) follows from (3.19), (3.22) and (3.23). 
We will also make use of the following elementary lemma from [1, Lemma 10].

Lemma 3.3 Let $\alpha, \beta$ and $\eta$ be positive constants. For $A>0$ and $B>0$, let

$$
J:=J(A, B)=\int_{0}^{1} \frac{d t}{\left(A+t^{\alpha}\right)^{\eta}(B+t)^{\beta}} .
$$

Then there exist finite constants $c_{3,9}$ and $c_{3,10}$, depending on $\alpha, \beta, \eta$ only, such that the following hold for all real numbers $A, B>0$ satisfying $A^{1 / \alpha} \leq c_{3,9} B$ :

(i) If $\alpha \eta>1$, then

$$
J \leq c_{3,10} \frac{1}{A^{\eta-\alpha^{-1} B^{\beta}}}
$$

(ii) If $0<\alpha \eta<1$ and $\alpha \eta+\beta \neq 1$, then

$$
J \leq c_{3,10}\left(\frac{1}{B^{\alpha \eta+\beta-1}}+1\right) .
$$

Now we are ready to prove Theorems 2.1, 2.3 and 2.5.

Proof of Theorem 2.1 We distinguish between three cases: (i) $d<Q$, (ii) $d=Q$ and (iii) $d>Q$. When $d<Q$, the second inequality in (2.5) holds automatically with $c_{2,8}=1$. Moreover, Theorem 7.1 in [23] implies that $X$ hits points, hence $\mathbb{P}\left\{X^{-1}(F) \cap I \neq \varnothing\right\}>0$ since $F \neq \varnothing$.

When $d>Q,(2.5)$ is proved in Theorem 7.6 in [23]. Hence it only remains to consider the case (ii). Again, the second inequality in (2.5) holds automatically with $c_{2,8}=1$.

The proof of the lower bound in (2.5) is similar to that of Theorem 7.6 in [23]. Without loss of generality, we assume $\mathcal{C}_{0}(F)>0$ otherwise there is nothing to prove. By Choquet's capacity theorem (cf. [12]), we may and will assume $F$ is compact and let $M>0$ be a constant such that $F \subseteq[-M, M]^{d}$.

By definition (1.3), there is a Borel probability measure $\nu_{0}$ on $F$ such that

$$
\mathcal{E}_{0}\left(\nu_{0}\right):=\int_{\mathbb{R}^{d}} \int_{\mathbb{R}^{d}} \log \left(\frac{e}{\|x-y\| \wedge 1}\right) \nu_{0}(d x) \nu_{0}(d y) \leq \frac{2}{\mathcal{C}_{0}(F)} .
$$

For all integers $n \geq 1$, we consider a family of random measures $\nu_{n}$ on $I$ defined by

$$
\begin{aligned}
\int_{I} g(t) \nu_{n}(d t) & =\int_{I} \int_{\mathbb{R}^{d}}(2 \pi n)^{d / 2} \exp \left(-\frac{n\|X(t)-x\|^{2}}{2}\right) g(t) \nu_{0}(d x) d t \\
& =\int_{I} \int_{\mathbb{R}^{d}} \int_{\mathbb{R}^{d}} \exp \left(-\frac{\|\xi\|^{2}}{2 n}+i\langle\xi, X(t)-x\rangle\right) g(t) d \xi \nu_{0}(d x) d t
\end{aligned}
$$

where $g$ is an arbitrary measurable, nonnegative function on $I$.

Denote the total mass of $\nu_{n}$ by $\left\|\nu_{n}\right\|:=\nu_{n}(I)$. We claim that the following two inequalities hold:

$$
\mathbb{E}\left(\left\|\nu_{n}\right\|\right) \geq c_{3,11} \quad \text { and } \quad \mathbb{E}\left(\left\|\nu_{n}\right\|^{2}\right) \leq c_{3,12} \mathcal{E}_{0}\left(\nu_{0}\right),
$$

where the constants $c_{3,11}$ and $c_{3,12}$ are independent of $\nu_{0}$ and $n$. 
Some simple calculation shows

$$
\begin{aligned}
\mathbb{E}\left(\left\|\nu_{n}\right\|\right) & =\int_{I} \int_{\mathbb{R}^{d}} \int_{\mathbb{R}^{d}} \exp \left(-\frac{1}{2}\left(\frac{1}{n}+\sigma^{2}(t)\right)\|\xi\|^{2}-i\langle\xi, x\rangle\right) d \xi \nu_{0}(d x) d t \\
& \geq \int_{I} \int_{\mathbb{R}^{d}} \frac{(2 \pi)^{d / 2}}{\left(1+\sigma^{2}(t)\right)^{d / 2}} \exp \left(-\frac{\|x\|^{2}}{2 \sigma^{2}(t)}\right) \nu_{0}(d x) d t \\
& \geq \int_{I} \frac{(2 \pi)^{d / 2}}{\left(1+\sigma^{2}(t)\right)^{d / 2}} \exp \left(-\frac{d M^{2}}{2 \sigma^{2}(t)}\right) d t:=c_{3,11},
\end{aligned}
$$

where $\sigma^{2}(t)=\mathbb{E}\left(X_{0}(t)^{2}\right)$ and the last inequality follows from the boundedness of $F$. Since $t \mapsto \sigma^{2}(t)$ is a positive continuous function on $I, c_{3,11}>0$. This gives the first inequality in $(3.29)$.

Denote by $I_{2 d}$ the identity matrix of order $2 d$ and let $\Gamma_{n}(s, t)=n^{-1} I_{2 d}+\operatorname{Cov}(X(s), X(t))$. Since $\Gamma_{n}(s, t)$ is positive definite, we have

$$
\begin{aligned}
& \mathbb{E}\left(\left\|\nu_{n}\right\|^{2}\right)=\int_{I} \int_{I} \int_{\mathbb{R}^{2 d}} \int_{\mathbb{R}^{2 d}} e^{-i(\langle\xi, x\rangle+\langle\eta, y\rangle)} \\
& \times \exp \left(-\frac{1}{2}(\xi, \eta) \Gamma_{n}(s, t)(\xi, \eta)^{\prime}\right) d \xi d \eta \nu_{0}(d x) \nu_{0}(d y) d s d t \\
& =\int_{I} \int_{I} \int_{\mathbb{R}^{2 d}} \frac{(2 \pi)^{d}}{\sqrt{\operatorname{det}\left(\Gamma_{n}(s, t)\right)}} \exp \left(-\frac{1}{2}(x, y) \Gamma_{n}^{-1}(s, t)(x, y)^{\prime}\right) \nu_{0}(d x) \nu_{0}(d y) d s d t \\
& \leq c_{3,13} \int_{I} \int_{I} \int_{\mathbb{R}^{2 d}} \frac{1}{\max \left\{\rho^{d}(s, t),\|x-y\|^{d}\right\}} \nu_{0}(d x) \nu_{0}(d y) d s d t,
\end{aligned}
$$

where the last inequality follows from Lemma 3.2 and the constant $c_{3,13}$ is independent of $\nu_{0}$ and $n$.

We claim that for all $x, y \in \mathbb{R}^{d}$,

$$
\int_{I} \int_{I} \frac{d s d t}{\max \left\{\rho^{d}(s, t),\|x-y\|^{d}\right\}} \leq c_{3,14} \log \left(\frac{e}{\|x-y\| \wedge 1}\right),
$$

where $c_{3,14}>0$ is a constant. To verify this, we break the integral in (3.32) over the regions $\{(s, t) \in I \times I: \rho(s, t) \leq\|x-y\|\}$ and $\{(s, t) \in I \times I: \rho(s, t)>\|x-y\|\}$ and denote them by $\mathcal{I}_{1}$ and $\mathcal{I}_{2}$, respectively. Since $d=Q$, we derive that

$$
\mathcal{I}_{1}=\int_{I} \int_{\{t \in I: \rho(s, t) \leq\|x-y\|\}} \frac{d s d t}{\|x-y\|^{d}} \leq \frac{c_{3,15}}{\|x-y\|^{d}}\|x-y\|^{Q}=c_{3,15} .
$$

In the above, we have used the fact that, for every $s \in I$, the set $\{t \in I: \rho(s, t) \leq\|x-y\|\}$ is contained in an rectangle of side-lengths $\|x-y\|^{1 / H_{j}}(j=1, \ldots, N)$.

On the other hand,

$$
\mathcal{I}_{2}=\int_{I} \int_{\{t \in I: \rho(s, t)>\|x-y\|\}} \frac{d s d t}{\rho^{d}(s, t)} \leq \int_{\left\{t \in[-1,1]^{N}: \rho(0, t)>\|x-y\|\right\}} \frac{d t}{\rho^{d}(0, t)}
$$

since $\rho(s, t)=\rho(0, t-s)$. Let us consider the diagonal matrix $E=\operatorname{diag}\left(1 / H_{1}, \ldots, 1 / H_{N}\right)$. Then, $t \mapsto \rho(0, t)$ is $E$-homogeneous function in the sense of Definition 2.6 of [4], that is

$$
\forall r>0, \quad \rho\left(0, r^{E} t\right)=r \rho(0, t)
$$


with $r^{E} \hat{=} \exp (\log (r) E)$. By using the formula of integration in the polar coordinates with respect to $E$ (see Proposition 2.3 in [15]) and $Q=d$, we have

$$
\mathcal{I}_{2} \leq \int_{0}^{+\infty} \int_{\mathcal{S}_{E}} \mathbf{1}_{\left\{r^{E} \theta \in[-1,1], r \rho(0, \theta)>\|x-y\|\right\}} \frac{\sigma_{E}(d \theta) d r}{r \rho^{d}(0, \theta)},
$$

where $\mathcal{S}_{E}$ is a compact set which does not contain 0 and $\sigma_{E}(d \theta)$ is a finite positive Radon measure on $\mathcal{S}_{E}$. Since

$$
0<\min _{\theta \in \mathcal{S}_{E}} \rho(0, \theta) \leq \max _{\theta \in \mathcal{S}_{E}} \rho(0, \theta)<\infty,
$$

there exist some finite positive constants $\ell, L, c, c_{3,16}$ such that

$$
\mathcal{I}_{2} \leq c \int_{0}^{L} \mathbf{1}_{\{r>\ell\|x-y\|\}} \frac{d r}{r} \leq c_{3,16} \log \left(\frac{e}{\|x-y\| \wedge 1}\right) .
$$

Combining (3.33) and (3.34) one verifies (3.32). It is clear that (3.31), (3.32) and Fubini's theorem imply the second inequality in (3.29).

By using (3.29) and an argument in [18, Lemma 2.9] or in [11, pp.204-206], one can verify that there is an event $\Omega_{0}$ with probability at least $c_{3,11}^{2} /\left(2 c_{3,12} \varepsilon_{0}\left(\nu_{0}\right)\right)$ such that for every $\omega \in \Omega_{0},\left\{\nu_{n}(\omega), n \geq 1\right\}$ has a subsequence that converges weakly to a finite positive measure $\nu$ which is supported on $X^{-1}(F) \cap I$. Then, we have

$$
\mathbb{P}\{X(I) \cap F \neq \varnothing\} \geq \mathbb{P}\{\|\nu\|>0\} \geq \frac{c_{3,11}^{2}}{2 c_{3,12} \varepsilon_{0}\left(\nu_{0}\right)} .
$$

Combining this with (3.27) yields the lower bound in (2.5). The proof of Theorem 2.1 is completed.

\section{Proof of Theorem 2.3}

Proof of Part (i) It is clear that the second statement in Part (i) follows from (2.7) directly. Hence we proceed to prove (2.7).

Since $\operatorname{dim}_{\mathrm{H}}$ is $\sigma$-stable ([8]), without loss of generality, we will assume that $F \subseteq[-M, M]^{d}$ for some constant $M>0$ to prove that (2.7) holds almost surely.

Let us choose and fix an arbitrary constant $\gamma>\operatorname{dim}_{\mathrm{H}} F$. Then, for $\delta_{0}$ in Lemma 3.1, there exists a constant $\delta \in\left(0, \delta_{0}\right)$ and a sequence of balls $\left\{B\left(x_{j}, r_{j}\right), j \geq 1\right\}$ in $\mathbb{R}^{d}$ (in Euclidean metric) such that $r_{j} \leq \delta$ for all $j \geq 1$,

$$
F \subseteq \bigcup_{j=1}^{\infty} B\left(x_{j}, r_{j}\right) \quad \text { and } \quad \sum_{j=1}^{\infty}\left(2 r_{j}\right)^{\gamma} \leq 1 .
$$

Moreover, we require $x_{j} \in[-M, M]^{d}$ for all $j \geq 1$.

For every integer $j \geq 1$, let $n_{j}$ be the integer such that $2^{-n_{j}-1} \leq r_{j}<2^{-n_{j}}$. We divide $I$ into sub-rectangles $R_{j, i}$ of side-lengths $2^{-n_{j} / H_{\ell}}(\ell=1, \ldots, N)$. Note that there are at most $c 2^{n_{j} Q}$ such rectangles (recall that $Q=\sum_{j=1}^{N} 1 / H_{j}$ ), and each $R_{j, i}$ is equivalent to a ball of radius $2^{-n_{j}}$ in the metric $\rho$. Let $N_{j}$ denote the number of $R_{j, i}$ 's such that $R_{j, i} \cap X^{-1}\left(B\left(x_{j}, r_{j}\right)\right) \neq \varnothing$. Hence, by using Lemma 3.1, we derive

$$
\mathbb{E}\left(N_{j}\right) \leq c_{3,17} 2^{n_{j} Q} 2^{-n_{j} d}=c_{3,17} 2^{n_{j}(Q-d)} .
$$


Since $X^{-1}(F) \subseteq \bigcup_{j=1}^{\infty} X^{-1}\left(B\left(x_{j}, r_{j}\right)\right)$, we have obtained a covering of $X^{-1}(F) \cap I$ by a subsequence of the rectangles $\left\{R_{j, i}\right\}$ (i.e., those satisfying $\left.R_{j, i} \cap X^{-1}\left(B\left(x_{j}, r_{j}\right)\right) \neq \varnothing\right)$.

For every fixed $k \in\{1, \ldots, N\}$, we see that each rectangle $R_{j, i}$ can be covered by at most $\prod_{\ell=k}^{N}\left(2^{n_{j}\left(1 / H_{k}-1 / H_{\ell}\right)}+1\right)$ cubes of side-length $2^{-n_{j} / H_{k}}$. In this way, we obtain a (random) covering of $X^{-1}(F) \cap I$ by cubes of side-length $2^{-n_{j} / H_{k}}$ which can be used to bound the Hausdorff measure of $X^{-1}(F) \cap I$. Let

$$
\beta_{k}=\sum_{j=1}^{k} \frac{H_{k}}{H_{j}}+N-k-H_{k}(d-\gamma) .
$$

It follows from (3.36) and (3.37) that

$$
\mathbb{E}\left[\sum_{j=1}^{\infty} N_{j} \prod_{\ell=k}^{N}\left(2^{n_{j}\left(\frac{1}{H_{k}}-\frac{1}{H_{\ell}}\right)}+1\right) 2^{-\frac{n_{j}}{H_{k}} \beta_{k}}\right] \leq c_{3,18} \sum_{j=1}^{\infty} 2^{-n_{j} \gamma} \leq c_{3,18} .
$$

This and Fatou's lemma together imply that $\mathcal{H}_{\beta_{k}}\left(X^{-1}(F) \cap I\right)<\infty$ almost surely. Hence we have $\operatorname{dim}_{\mathrm{H}}\left(X^{-1}(F) \cap I\right) \leq \beta_{k}$ a.s. for every $k \in\{1, \ldots, N\}$. Letting $\gamma \downarrow \operatorname{dim}_{\mathrm{H}} F$ along rational numbers yields $(2.7)$.

Proof of Part (ii) Assume that $F$ is a non-empty set such that $\operatorname{dim}_{\mathrm{H}} F>d-Q$. Note that if $\operatorname{dim}_{\mathrm{H}} F=0$, then we have $\sum_{j=1}^{N} 1 / H_{j}>d$. For any fixed $x \in F$, we have $X^{-1}(F) \supseteq X^{-1}(\{x\})$ and (2.9) follows from Theorem 7.1 in [23] and (2.7). It remains to consider those Borel sets $F \subseteq \mathbb{R}^{d}$ with $\operatorname{dim}_{\mathrm{H}} F>0$.

For this purpose, let us note that (2.6) implies

$$
\begin{aligned}
& \min _{1 \leq k \leq N}\left\{\sum_{j=1}^{k} \frac{H_{k}}{H_{j}}+N-k-H_{k}\left(d-\operatorname{dim}_{\mathrm{H}} F\right)\right\} \\
& =\sum_{j=1}^{k} \frac{H_{k}}{H_{j}}+N-k-H_{k}\left(d-\operatorname{dim}_{\mathrm{H}} F\right), \quad \text { if } \sum_{j=1}^{k-1} \frac{1}{H_{j}} \leq d-\operatorname{dim}_{\mathrm{H}} F<\sum_{j=1}^{k} \frac{1}{H_{j}} .
\end{aligned}
$$

The verification of (3.39) is elementary (cf. Lemma 7 in [1]) and is omitted.

Let $k \in\{1, \ldots, N\}$ be the unique integer satisfying

$$
\sum_{j=1}^{k-1} \frac{1}{H_{j}} \leq d-\operatorname{dim}_{\mathrm{H}} F<\sum_{j=1}^{k} \frac{1}{H_{j}} .
$$

By (3.39), it is sufficient to prove that for every $\varepsilon>0$, the inequality

$$
\operatorname{dim}_{\mathrm{H}}\left(X^{-1}(F) \cap I\right) \geq \sum_{j=1}^{k} \frac{H_{k}}{H_{j}}+N-k-H_{k}\left(d-\operatorname{dim}_{\mathrm{H}} F\right)-\varepsilon
$$

holds on an event with positive probability (which may depend on $\varepsilon$ ).

Let us choose and fix an arbitrary constant $\delta \in\left(0, \operatorname{dim}_{\mathrm{H}} F\right)$ such that

$$
\sum_{j=1}^{k-1} \frac{1}{H_{j}}<d-\operatorname{dim}_{\mathrm{H}} F+\delta<\sum_{j=1}^{k} \frac{1}{H_{j}}
$$


(this is possible because of (3.40)) and define

$$
\beta_{\delta}:=\sum_{j=1}^{k} \frac{H_{k}}{H_{j}}+N-k-H_{k}\left(d-\operatorname{dim}_{\mathrm{H}} F\right)-H_{k} \delta .
$$

Then it can be verified that $\beta_{\delta} \in(N-k, N-k+1)$, and (3.41) follows from

$$
\operatorname{dim}_{\mathrm{H}}\left(X^{-1}(F) \cap I\right) \geq \beta_{\delta}
$$

on an event with positive probability (which may depend on $\delta$ ).

Now we prove (3.44). Let $\gamma \in\left(\operatorname{dim}_{\mathrm{H}} F-\delta, \operatorname{dim}_{\mathrm{H}} F\right)$ be a constant. Then (3.42) still holds when $\operatorname{dim}_{\mathrm{H}} F-\delta$ is replaced by $\gamma$. Then there is a compact set $F_{\gamma} \subseteq F$ such that $\operatorname{dim}_{\mathrm{H}} F_{\gamma}>\gamma$ (see [8]). It follows from Frostman's lemma (cf. [8] or [11]) that there exists a Borel probability measure $\mu_{0, \gamma}$ supported on $F_{\gamma}$ such that

$$
\mu_{0, \gamma}(B(x, r)) \leq c_{3,19} r^{\gamma} \quad \text { for all } x \in \mathbb{R}^{d} \text { and } r>0,
$$

where $c_{3,19}$ may depend on $\gamma$.

We construct a sequence $\left\{\mu_{n, \gamma}, n \geq 1\right\}$ of random Borel measures on $I$ as in (3.28) with $\nu_{0}$ replaced by $\mu_{0, \gamma}$. We will prove the following statements:

(i) There exist finite positive constants $c_{3,20}$ and $c_{3,21}$ (which may depend on $\gamma$ ) such that

$$
\mathbb{E}\left(\left\|\mu_{n, \gamma}\right\|\right) \geq c_{3,20} \quad \text { and } \quad \mathbb{E}\left(\left\|\mu_{n, \gamma}\right\|^{2}\right) \leq c_{3,21}
$$

hold for all integers $n \geq 1$.

(ii) For the constant $\beta_{\delta}$ defined by (3.43), there exists a finite positive constant $c_{3,22}$ such that for all $n \geq 1$

$$
\mathbb{E} \int_{I} \int_{I} \frac{1}{\|s-t\|^{\beta_{\delta}}} \mu_{n, \gamma}(d s) \mu_{n, \gamma}(d t) \leq c_{3,22} .
$$

That is, the expected $\beta_{\delta}$-energy of the random measures $\left\{\mu_{n, \gamma}\right\}$ are bounded.

The first inequality in (3.46) is derived in the same way as (3.30), because the probability measure $\mu_{0, \gamma}$ is supported on the compact set $F_{\gamma}$. Now we prove the second inequality in (3.46). Similar to (3.31), we use Lemma 3.2 to obtain

$$
\mathbb{E}\left(\left\|\mu_{n, \gamma}\right\|^{2}\right) \leq c_{3,23} \int_{I} \int_{I} \int_{\mathbb{R}^{2 d}} \frac{1}{\max \left\{\rho^{d}(s, t),\|x-y\|^{d}\right\}} \mu_{0, \gamma}(d x) \mu_{0, \gamma}(d y) d s d t .
$$

By property (3.45) of $\mu_{0, \gamma}$, it can be shown that

$$
\int_{\mathbb{R}^{2 d}} \frac{1}{\max \left\{\rho^{d}(s, t),\|x-y\|^{d}\right\}} \mu_{0, \gamma}(d x) \mu_{0, \gamma}(d y) \leq \frac{c_{3,24}}{\rho(s, t)^{d-\gamma}} .
$$

To this end, we break the above integral over $\left\{(x, y) \in \mathbb{R}^{2 d}:\|x-y\| \geq \rho(s, t)\right\}$ and $\{(x, y) \in$ $\left.\mathbb{R}^{2 d}:\|x-y\|<\rho(s, t)\right\}$ and write them as $\mathcal{J}_{1}$ and $\mathcal{J}_{2}$, respectively. For each fixed $x \in \mathbb{R}^{d}$, let $\kappa_{x}$ be the image measure of $\mu_{0, \gamma}$ under the mapping $T: y \mapsto\|x-y\|$ from $\mathbb{R}^{d}$ to $\mathbb{R}_{+}$.

$$
\begin{aligned}
\mathcal{J}_{1} & =\int_{\mathbb{R}^{d}} \mu_{0, \gamma}(d x) \int_{\{\|x-y\| \geq \rho(s, t)\}} \frac{\mu_{0, \gamma}(d y)}{\|x-y\|^{d}}=\int_{\mathbb{R}^{d}} \mu_{0, \gamma}(d x) \int_{\rho(s, t)}^{+\infty} \frac{1}{r^{d}} \kappa_{x}(d r) \\
& \leq d c_{3,19} \int_{\mathbb{R}^{d}} \mu_{0, \gamma}(d x) \int_{\rho(s, t)}^{+\infty} r^{\gamma-d-1} d r=\frac{c_{3,25}}{\rho(s, t)^{d-\gamma}}
\end{aligned}
$$


where the above inequality follows from an integration-by-parts formula and (3.45).

On the other hand, (3.45) implies that

$$
\mathcal{J}_{2}=\int_{\mathbb{R}^{d}} \mu_{0, \gamma}(d x) \int_{\{\|x-y\|<\rho(s, t)\}} \frac{\mu_{0, \gamma}(d y)}{\rho(s, t)^{d}} \leq \frac{c_{3,19}}{\rho(s, t)^{d-\gamma}} .
$$

Hence (3.49) follows from (3.50) and (3.51).

It follows from (3.48), (3.49), Condition (C2) and some elementary computation that

$$
\mathbb{E}\left(\left\|\mu_{n, \gamma}\right\|^{2}\right) \leq c_{3,26} \int_{I} \int_{I} \frac{1}{\left[\sum_{j=1}^{N}\left|s_{j}-t_{j}\right|^{H_{j}}\right]^{d-\gamma}} d s d t:=c_{3,21}<\infty .
$$

In the above, we have used (3.42) to show the last integral is convergent (cf. [1], p. 432). Thus we have verified the second inequality in (3.46).

Similar to (3.48)-(3.52) and recall that $I=\left[\varepsilon_{0}, 1\right]^{N}$, we derive

$$
\begin{aligned}
& \mathbb{E} \int_{I} \int_{I} \frac{1}{\|s-t\|^{\beta_{\delta}}} \mu_{n, \gamma}(d s) \mu_{n, \gamma}(d t) \\
& \leq c_{3,27} \int_{I} \int_{I} \frac{1}{\left[\sum_{j=1}^{N}\left|s_{j}-t_{j}\right|^{\left.H_{j}\right]^{d-\gamma}}\left(\sum_{j=1}^{N}\left|s_{j}-t_{j}\right|\right)^{\beta_{\delta}}\right.} d s d t \\
& \leq c_{3,28} \int_{[0,1]^{N}} \frac{1}{\left[\sum_{j=1}^{N} t_{j}^{H_{j}}\right]^{d-\gamma}\left(\sum_{j=1}^{N} t_{j}\right)^{\beta_{\delta}}} d t .
\end{aligned}
$$

Let us denote the last integral in $(3.53)$ by $\mathcal{G}$ and apply Lemma 3.3 to verify that it is convergent. Without loss of generality, we assume $k \geq 2$, otherwise we jump to (3.56) directly. We integrate $\left[d t_{1}\right]$ first. By $(3.42)$ we see that $H_{1}(d-\gamma)>1$. Hence we can use (3.25) of Lemma 3.3 with $\alpha=H_{1}, \eta=d-\gamma, A=\sum_{j=2}^{N} t_{j}^{H_{j}}$ and $B=\sum_{j=2}^{N} t_{j}$ to get

$$
\mathcal{G} \leq c_{3,29} \int_{0}^{1} d t_{N} \cdots \int_{0}^{1} \frac{1}{\left(\sum_{j=2}^{N} t_{j}^{H_{j}}\right)^{d-\gamma-1 / H_{1}}\left(\sum_{j=2}^{N} t_{j}\right)^{\beta_{\delta}}} d t_{2} .
$$

We repeat this procedure for integrating $d t_{2}, \ldots, d t_{k-1}$ to obtain

$$
\mathcal{G} \leq c_{3,30} \int_{0}^{1} d t_{N} \cdots \int_{0}^{1} \frac{d t_{k}}{\left(\sum_{j=k}^{N} t_{j}^{H_{j}}\right)^{d-\gamma-\sum_{j=1}^{k-1} 1 / H_{j}}\left(\sum_{j=k}^{N} t_{j}\right)^{\beta_{\delta}}} .
$$

To integrate $\left[d t_{k}\right]$ in $(3.55)$, note that $H_{k}\left(d-\gamma-\sum_{j=1}^{k-1} 1 / H_{j}\right)<1$. It follows from (3.26) that

$$
\begin{aligned}
\mathcal{G} & \leq c_{3,31}\left[\int_{0}^{1} d t_{N} \cdots \int_{0}^{1} \frac{d t_{k+1}}{\left(\sum_{j=k+1}^{N} t_{j}\right)^{H_{k}\left(d-\gamma-\sum_{j=1}^{k-1} 1 / H_{j}\right)+\beta_{\delta}-1}}+1\right] \\
& =c_{3,31}\left[\int_{0}^{1} d t_{N} \cdots \int_{0}^{1} \frac{d t_{k+1}}{\left.\left(\sum_{j=k+1}^{N} t_{j}\right)^{N-k+H_{k}\left(\operatorname{dim}_{\mathrm{H}} F-\delta-\gamma\right)}+1\right] .}\right.
\end{aligned}
$$

Since $\operatorname{dim}_{\mathrm{H}} F-\delta<\gamma$, the last integral is convergent. Hence (3.47) holds.

By using (3.46), (3.47) and the argument in [18, Lemma 2.9] or in [11, pp.204-206] again, one can verify that there is an event $\Omega_{\gamma}$ with probability at least $c_{3,20}^{2} /\left(2 c_{3,21}\right)$ such that, for 
every $\omega \in \Omega_{\gamma},\left\{\mu_{n, \gamma}(\omega), n \geq 1\right\}$ has a subsequence $\left\{\mu_{n_{k}, \gamma}(\omega), k \geq 1\right\}$ that converges weakly to a positive measure $\mu_{\gamma}=\mu_{\gamma}(\omega)$, which is supported on $X^{-1}(F) \cap I$. Moreover, the $\beta_{\delta}$-energy of $\left\{\mu_{n_{k}, \gamma}(\omega), k \geq 1\right\}$ are bounded. This and the monotone convergence theorem together imply that, on the event $\Omega_{\gamma}$, the $\beta_{\delta}$-energy of $\mu_{\gamma}$ is finite. Therefore by Frostman's theorem (cf. [8] or [11]) we derive that (3.41) holds on an event with probability at least $c_{3,20}^{2} /\left(2 c_{3,21}\right)$. This finishes the proof of Theorem 2.3.

Finally we show that, under the extra condition $(S)$, the proof of Theorem 2.3 can be strengthened to prove Theorem 2.5.

Proof of Theorem 2.5 The second equality in (2.14) is implied by (3.39). Hence it only remains to prove that, if $F \subseteq \mathbb{R}^{d}$ is a Borel set satisfying

$$
\sum_{j=1}^{k-1} \frac{1}{H_{j}} \leq d-\operatorname{dim}_{\mathrm{H}} F<\sum_{j=1}^{k} \frac{1}{H_{j}}
$$

for some integer $k \in\{1, \ldots, N\}$, then

$$
\operatorname{dim}_{\mathrm{H}}\left(X^{-1}(F) \cap I\right) \geq \sum_{j=1}^{k} \frac{H_{k}}{H_{j}}+N-k-H_{k}\left(d-\operatorname{dim}_{\mathrm{H}} F\right)
$$

holds with positive probability. We will prove the following statement: There exist constants $\delta_{0} \in\left(0, \operatorname{dim}_{\mathrm{H}} F\right)$ and $c_{3,32}>0$ such that for all $\delta \in\left(0, \delta_{0}\right)$ we have

$$
\sum_{j=1}^{k-1} \frac{1}{H_{j}}<d-\operatorname{dim}_{\mathrm{H}} F+\delta<\sum_{j=1}^{k} \frac{1}{H_{j}}
$$

and

$$
\operatorname{dim}_{\mathrm{H}}\left(X^{-1}(F) \cap I\right) \geq \sum_{j=1}^{k} \frac{H_{k}}{H_{j}}+N-k-H_{k}\left(d-\operatorname{dim}_{\mathrm{H}} F\right)-H_{k} \delta
$$

holds with probability greater than $c_{3,32}$ (which does not depend on $\delta$ ). Letting $\delta \downarrow 0$, this shows that (3.58) holds with probability greater than $c_{3,32}$.

The proof of the above statement is almost the same as the proof of (3.44). Since (3.57) is fulfilled, we can choose $\delta_{0} \in\left(0, \operatorname{dim}_{\mathrm{H}} F\right)$ such that (3.59) holds for all $\delta \in\left(0, \delta_{0}\right)$.

Let $\delta \in\left(0, \delta_{0}\right), \gamma \in\left(\operatorname{dim}_{\mathrm{H}} F-\delta, \operatorname{dim}_{\mathrm{H}} F\right)$ and define $\beta_{\delta}$ by (3.43). Let $\mu_{0, \gamma}$ be the probability measure with compact support in $F$ given by Condition $(S)$, and let $\left\{\mu_{n, \gamma}, n \geq 1\right\}$ be the sequence of random Borel measures on $I$ constructed in the proof of Theorem 2.3. The only difference is that we will show the constants $c_{3,20}$ and $c_{3,21}$ in (3.46) are independent of $\delta$ and $\gamma$.

Since $F$ is bounded, we derive as in (3.30) that $c_{3,20}$ is independent of $\mu_{0, \gamma}, \gamma$ and $\delta$. Next we prove that $\mathbb{E}\left(\left\|\mu_{n, \gamma}\right\|^{2}\right) \leq c_{3,21}$ for all $\gamma \in\left(\operatorname{dim}_{\mathrm{H}} F-\delta, \operatorname{dim}_{\mathrm{H}} F\right)$, and the constant $c_{3,21}$ only depends on $F, \delta_{0}, c_{2,9}, I$ and $H$.

Recall from (3.48) that

$$
\mathbb{E}\left(\left\|\mu_{n, \gamma}\right\|^{2}\right) \leq c_{3,23} \int_{I} \int_{I} \int_{\mathbb{R}^{2 d}} \frac{1}{\max \left\{\rho^{d}(s, t),\|x-y\|^{d}\right\}} \mu_{0, \gamma}(d x) \mu_{0, \gamma}(d y) d s d t
$$


Since $\gamma>\operatorname{dim}_{\mathrm{H}} F-\delta_{0}>0$ and $c_{2,9} \geq 1$, we see from (2.11) that the probability measure $\mu_{0, \gamma}$ also satisfies

$$
\mu_{0, \gamma}(B(x, r)) \leq c_{2,9} r^{\operatorname{dim}_{\mathrm{H}} F-\delta_{0}} \quad \text { for all } x \in \mathbb{R}^{d} \text { and } r \in(0, \infty) .
$$

Similar to the verification of (3.49), we derive from (3.62) that

$$
\int_{\mathbb{R}^{2 d}} \frac{1}{\max \left\{\rho^{d}(s, t),\|x-y\|^{d}\right\}} \mu_{0, \gamma}(d x) \mu_{0, \gamma}(d y) \leq \frac{c_{3,33}}{\rho(s, t)^{d-\operatorname{dim}_{\mathrm{H}} F+\delta_{0}}} .
$$

Here the constant $c_{3,33}$ depends only on $d, F, c_{2,9}$ and $\delta_{0}$. It follows from (3.61) and (3.63) that

$$
\mathbb{E}\left(\left\|\mu_{n, \gamma}\right\|^{2}\right) \leq c_{3,34} \int_{I} \int_{I} \frac{1}{\left[\sum_{j=1}^{N}\left|s_{j}-t_{j}\right|^{H_{j}}\right]^{d-\operatorname{dim}_{\mathrm{H}} F+\delta_{0}}} d s d t:=c_{3,21}<\infty .
$$

This verifies the second inequality in (3.46) and the constant $c_{3,21}$ is independent of $\gamma$ and $\delta$.

Observe that (3.47) remains valid for $\mu_{n, \gamma}$, and the constant $c_{3,22}$ does not affect the lower bound for $\mathbb{P}\left(\Omega_{\gamma}\right)$ in the proof of Theorem 2.3. The same proof shows the existence of an event $\Omega_{\gamma}$ of probability at least $c_{3,20}^{2} /\left(2 c_{3,21}\right)$ such that, for every $\omega \in \Omega_{\gamma},\left\{\mu_{n, \gamma}(\omega), n \geq 1\right\}$ has a subsequence that converges weakly to a positive measure $\mu_{\gamma}$, which is supported on $X^{-1}(F) \cap I$ and has finite $\beta_{\delta}$-energy.

Define $c_{3,32}:=c_{3,20}^{2} /\left(2 c_{3,21}\right)$. Then the constant $c_{3,32}$ does not depend on $\delta$. It follows from the above and Frostman's theorem that for all $\delta \in\left(0, \delta_{0}\right)$

$$
\operatorname{dim}_{\mathrm{H}}\left(X^{-1}(F) \cap I\right) \geq \beta_{\delta}
$$

on an event with probability greater than $c_{3,32}$. This verifies (3.60) and the proof of Theorem 2.5 is finished.

\section{Applications to stochastic partial differential equations}

Theorems 2.1, 2.3 and 2.5 are applicable to solutions of stochastic partial differential equations (SPED) such as linear hyperbolic SPDE considered by Dalang and Nualart [7] and nonlinear stochastic heat equations considered by Dalang, Khoshnevisan and Nualart [5]. In this section we only consider the Hausdorff dimension of the inverse images of nonlinear stochastic heat equations in [5].

Let $\dot{W}=\left(\dot{W}_{1}, \ldots, \dot{W}_{d}\right)$ be a space-time white noise in $\mathbb{R}^{d}$. That is, the components $\dot{W}_{1}(x, t), \ldots, \dot{W}_{d}(x, t)$ of $\dot{W}(x, t)$ are independent space-time white noises, which are generalized Gaussian processes with covariance given by

$$
\mathbb{E}\left[\dot{W}_{i}(t, x) \dot{W}_{i}(y, s)\right]=\delta(x-y) \delta(t-s), \quad(i=1, \ldots, d),
$$

where $\delta(\cdot)$ is the Dirac delta function. For all $1 \leq j \leq d$, let $b_{j}: \mathbb{R}^{d} \rightarrow \mathbb{R}$ be globally Lipschitz and bounded functions, and let $\sigma:=\left(\sigma_{i, j}\right)$ be a deterministic $d \times d$ invertible matrix.

Consider the system of stochastic partial differential equations

$$
\frac{\partial u_{i}}{\partial t}(t, x)=\frac{\partial^{2} u_{i}}{\partial x^{2}}(t, x)+\sum_{j=1}^{d} \sigma_{i, j} \dot{W}_{j}(t, x)+b_{i}(u(t, x))
$$


for $1 \leq i \leq d, t \in[0, T]$ and $x \in[0,1]$, with the initial conditions $u(0, x)=0$ for all $x \in[0,1]$, and the Neumann boundary conditions

$$
\frac{\partial u_{i}}{\partial x}(t, 0)=\frac{\partial u_{i}}{\partial x}(t, 1)=0, \quad 0 \leq t \leq T .
$$

In the above, $u(t, x)=\left(u_{1}(t, x), \ldots, u_{d}(t, x)\right)$.

Following Dalang, Khoshnevisan and Nualart [5] (see also Walsh [19]), Equation (4.1) can be interpreted rigorously as follows. Let $W_{i}=\left\{W_{i}(s, x), s \in \mathbb{R}_{+}, x \in[0,1]\right\}(1 \leq i \leq d)$ be independent Brownian sheets, defined on a probability space $(\Omega, \mathcal{F}, \mathbb{P})$, and set $W=$ $\left(W_{1}, \ldots, W_{d}\right)$. For $t \geq 0$, let $\mathcal{F}_{t}=\sigma(W(s, x): s \in[0, t], x \in[0,1])$. We say that a random field $u=\{u(t, x), t \in[0, T], x \in[0,1]\}$ is adapted to $\left(\mathcal{F}_{t}\right)$ if $u(t, x)$ is $\mathcal{F}_{t^{-}}$-measurable for every $(t, x) \in[0, T] \times[0,1]$. A random field $u=\{u(t, x), t \in[0, T], x \in[0,1]\}$ is a solution of (4.1) if $u$ is adapted to $\left(\mathcal{F}_{t}\right)$ and if for every $i \in\{1, \ldots, d\}, t \in[0, T]$ and $x \in[0,1]$,

$$
u_{i}(t, x)=\int_{0}^{t} \int_{0}^{1} G_{t-r}(x, v) \sum_{j=1}^{d} \sigma_{i, j} W_{j}(d r, d v)+\int_{0}^{t} \int_{0}^{1} G_{t-r}(x, v) b_{i}(u(t, x)) d r d v
$$

where $G_{t}(x, y)$ is the Green kernel for the heat equation with Neumann boundary conditions (see Walsh [19]).

For the linear form of (4.1) [i.e., $b \equiv 0$ and $\sigma \equiv I_{d}$ (the $d \times d$ identity matrix)], Mueller and Tribe [16] found necessary and sufficient conditions (in terms of the dimension $d$ ) for its solution $u$ to hit points or to have double points of various types. Wu and Xiao [20] further studied the fractal properties of the sample paths of $u$. In particular, they obtained the Hausdorff dimensions of the level sets and the set of double times of $u$.

More generally, Dalang, Khoshnevisan and Nualart [5] studied hitting probabilities for the non-linear equation (4.1) by providing sufficient conditions and necessary conditions for a Borel set $F \subseteq \mathbb{R}^{d}$ to be polar for the processes $\{u(t, x), t \in[0, T], x \in[0,1]\},\{u(t, x), x \in$ $[0,1]\}(t \in(0, T]$ is fixed) and $\{u(t, x), t \in[0, T]\} \quad(x \in[0,1]$ is fixed). They also determined the Hausdorff dimensions of the range and level sets of these processes.

In the following, we show that the hitting probability results of Dalang, Khoshnevisan and Nualart [5] can be derived from Theorem 2.1. Moreover, we apply Theorems 2.3 and 2.5 to further determine the Hausdorff dimension of inverse images of the processes $u=\{u(t, x), t \in$ $[0, T], x \in[0,1]\}, u_{t}=\{u(t, x), x \in[0,1]\}(t \in(0, T]$ is fixed $)$ and $u_{x}=\{u(t, x), t \in[0, T]\}$ $(x \in[0,1]$ is fixed). As shown by Proposition 5.1 in [5], it is sufficient to consider these problems for the solution of equation (4.1) in the following drift-free case [i.e., $b \equiv 0$ ]:

$$
\frac{\partial u}{\partial t}(t, x)=\frac{\partial^{2} u}{\partial x^{2}}(t, x)+\sigma \dot{W}
$$

The solution of (4.4) is the mean zero Gaussian random field $u=\{u(t, x), t \in[0, T], x \in[0,1]\}$ with values in $\mathbb{R}^{d}$ defined by

$$
u(t, x)=\int_{0}^{t} \int_{0}^{1} G_{t-r}(x, y) \sigma W(d r, d y), \quad \forall t \in[0, T], x \in[0,1] .
$$

Moreover, since the matrix $\sigma$ is invertible, a change of variables shows (see the proof of Proposition 4.1 in [5]) that $v:=\sigma^{-1} u$ solves the following uncoupled system of SPDE

$$
\frac{\partial v}{\partial t}(t, x)=\frac{\partial^{2} v}{\partial x^{2}}(t, x)+\dot{W}
$$


Note that both processes $u$ and $v$ have the same hitting probability and Hausdorff dimension properties. Therefore, without loss of generality, we will assume that $\sigma=I_{d}$ in (4.4).

The following is a consequence of Lemmas 4.2 and 4.3 of Dalang, Khoshnevisan and Nualart [5].

Lemma 4.1 Let $u=\{u(t, x), t \in[0, T], x \in[0,1]\}$ be the solution of (4.4). Then for every $t_{0} \in(0, T)$, there exist positive and finite constants $c_{4,1}, \ldots, c_{4,5}$ such that the following hold:

(i) For all $t \in I=\left[t_{0}, T\right] \times[0,1]$, we have $c_{4,1} \leq \mathbb{E}\left[u(t, x)^{2}\right] \leq c_{4,2}$ and

$$
\begin{aligned}
c_{4,3}\left(|t-s|^{1 / 4}+|x-y|^{1 / 2}\right)^{2} \leq \mathbb{E}[ & \left.(u(t, x)-u(s, y))^{2}\right] \\
& \leq c_{4,4}\left(|t-s|^{1 / 4}+|x-y|^{1 / 2}\right)^{2} .
\end{aligned}
$$

(ii) For all $(t, x),(s, y) \in I$,

$$
\operatorname{Var}(u(t, x) \mid u(s, y)) \geq c_{4,5}\left(|t-s|^{1 / 4}+|x-y|^{1 / 2}\right)^{2} .
$$

In other words, Lemma 4.1 states that the Gaussian random field $u$ satisfies Conditions (C1) and (C2) with $H_{1}=1 / 4$ and $H_{2}=1 / 2$.

Proof It follows from (4.5) that

$$
\mathbb{E}\left[u(t, x)^{2}\right]=\int_{0}^{t} d r \int_{0}^{1}\left(G_{t-r}(x, v)\right)^{2} d v .
$$

It can be verified that the function $\sigma^{2}(t, x):=\mathbb{E}\left[u(t, x)^{2}\right]$ is continuous in $(t, x)$ and positive on $I$. This implies the first conclusion of the lemma. The inequality (4.7) is the same as (4.11) in Lemma 4.2 of Dalang, Khoshnevisan and Nualart [5].

To prove (4.8), we note that Lemma 4.3 of Dalang, Khoshnevisan and Nualart [5] shows that

$$
\operatorname{det} \operatorname{Cov}(u(t, x), u(s, y)) \geq c_{4,6}\left(|t-s|^{1 / 4}+|x-y|^{1 / 2}\right)^{2} .
$$

Hence (4.8) follows from (3.16), (4.9) and the fact that $\mathbb{E}\left[u(s, y)^{2}\right] \leq c_{4,2}$ for all $t \in I$. This proves Lemma 4.1.

Remark 4.2 Lemma 4.1 implies that, for every fixed $t \in\left[t_{0}, T\right]$, the Gaussian process $u_{t}=\{u(t, x), x \in[0,1]\}$ has the following properties:

$$
\begin{gathered}
c_{4,1} \leq \mathbb{E}\left[u_{t}(x)^{2}\right] \leq c_{4,2}, \quad \forall x \in[0,1], \\
c_{4,3}|x-y| \leq \mathbb{E}\left[\left(u_{t}(x)-u_{t}(y)\right)^{2}\right] \leq c_{4,4}|x-y|, \quad \forall x, y \in[0,1]
\end{gathered}
$$

and

$$
\operatorname{Var}\left(u_{t}(x) \mid u_{t}(y)\right) \geq c_{4,5}|x-y|, \quad \forall x, y \in[0,1] .
$$

This shows that the Gaussian process $u_{t}$ satisfies the two-point local nondeterminism with index $H_{2}=1 / 2$. Similarly, for every fixed $x \in(0,1]$, the Gaussian process $u_{x}=\{u(t, x), t \in$ $\left.\left[t_{0}, T\right]\right\}$ satisfies the two-point local nondeterminism with $H_{1}=1 / 4$.

Hence we can apply Theorem 2.1 to recover the results of Dalang, Khoshnevisan and Nualart [5] on the hitting probabilities of the solution to (4.1). The following theorem, which extends the Hausdorff dimension results in [5], is a consequence of Theorems 2.3 and 2.5. 
Theorem 4.3 Let $u=\{u(t, x), t \in[0, T], x \in[0,1]\}$ denote the solution to (4.1) and let $F \subseteq \mathbb{R}^{d}$ be a Borel set. The following conclusions hold:

(i) If $\operatorname{dim}_{\mathrm{H}} F \geq d-6$, then

$$
\left\|\operatorname{dim}_{\mathrm{H}}\left(u^{-1}(F) \cap I\right)\right\|_{L^{\infty}(\mathbb{P})}= \begin{cases}2-\frac{1}{4}\left(d-\operatorname{dim}_{\mathrm{H}} F\right) & \text { if } 0 \leq d-\operatorname{dim}_{\mathrm{H}} F<4, \\ 3-\frac{1}{2}\left(d-\operatorname{dim}_{\mathrm{H}} F\right) & \text { if } 4 \leq d-\operatorname{dim}_{\mathrm{H}} F \leq 6 .\end{cases}
$$

(ii) If $\operatorname{dim}_{\mathrm{H}} F \geq d-2$, then for every fixed $t \geq t_{0}$ we have

$$
\left\|\operatorname{dim}_{\mathrm{H}}\left(u_{t}^{-1}(F) \cap[0,1]\right)\right\|_{L^{\infty}(\mathbb{P})}=1-\frac{1}{2}\left(d-\operatorname{dim}_{\mathrm{H}} F\right) .
$$

(iii) If $\operatorname{dim}_{\mathrm{H}} F \geq d-4$, then for every fixed $x \in[0,1]$ we have

$$
\left\|\operatorname{dim}_{\mathrm{H}}\left(u_{x}^{-1}(F) \cap[0, T]\right)\right\|_{L^{\infty}(\mathbb{P})}=1-\frac{1}{4}\left(d-\operatorname{dim}_{\mathrm{H}} F\right) .
$$

If, in addition, $F$ is bounded and satisfies Condition $(S)$, then the equalities without $\|\cdot\|_{L^{\infty}(\mathbb{P})}$ in (4.13)-(4.15) hold with positive probability.

Proof As we mentioned earlier, it is sufficient to prove the results for the case of $b \equiv 0$ and $\sigma=I_{d}$ in (4.1). Therefore, the conclusions follow from Theorem 2.3, Theorem 2.5, Lemma 4.1 and Remark 4.2 .

\section{References}

[1] A. Ayache and Y. Xiao (2005), Asymptotic properties and Hausdorff dimensions of fractional Brownian sheets. J. Fourier Anal. Appl. 11, 407-439.

[2] H. Biermé, A. Estrade, M. M. Meerschaert and Y. Xiao (2008), Sample path properties of operator scaling Gaussian random fields. In Preparation.

[3] H. Biermé and C. Lacaux (2007), Hölder regularity for operator scaling stable random fields. Submitted.

[4] H. Biermé, M. M. Meerschaert and H.-P. Scheffler (2007), Operator scaling stable random fields. Stoch. Process. Appl. 117, 312-332.

[5] R. C. Dalang, D. Khoshnevisan and E. Nualart (2007a), Hitting probabilities for systems of non-linear stochastic heat equations with additive noise. Latin Amer. J. Probab. Statist. (Alea) 3, 231-271.

[6] R. C. Dalang, D. Khoshnevisan and E. Nualart (2007b), Hitting probabilities for the non-linear stochastic heat equation with multiplicative noise. Submitted.

[7] R. C. Dalang and E. Nualart (2004), Potential theory for hyperbolic SPDEs. Ann. Probab. 32, 2099-2148.

[8] K. J. Falconer (1990), Fractal Geometry - Mathematical Foundations and Applications. John Wiley \& Sons Ltd., Chichester.

[9] J. Hawkes (1971), On the Hausdorff dimension of the intersection of the range of a stable process with a Borel set. Z. Wahrsch. Verw. Gebiete 19, 90-102. 
[10] A. Jonsson and H. Wallin (1984), Function Spaces on Subsets of $\mathbb{R}^{n}$. Harwood Academic Publishers, London.

[11] J.-P. Kahane (1985), Some Random Series of Functions. 2nd edition, Cambridge University Press, Cambridge.

[12] D. Khoshnevisan (2002), Multiparameter Processes: An Introduction to Random Fields. Springer, New York.

[13] D. Khoshnevisan and Y. Xiao (2005), Lévy processes: capacity and Hausdorff dimension. Ann. Probab. 33, 841-878.

[14] D. Khoshnevisan and Z. Shi (1999), Brownian sheet and capacity. Ann. Probab. 27, 1135-1159.

[15] M. M. Meerschaert and H. P. Scheffler (2001), Limit Distributions for Sums of Independent Random Vectors. John Wiley \& Sons Inc., New York.

[16] C. Mueller and R. Tribe (2002), Hitting probabilities of a random string. Electron. J. Probab. 7, Paper No. 10, 1-29.

[17] D. Monrad and L. D. Pitt (1987), Local nondeterminism and Hausdorff dimension. Progress in Probability and Statistics. Seminar on Stochastic Processes 1986, (E, Cinlar, K. L. Chung, R. K. Getoor, Editors), pp. 163-189, Birkhäuser, Boston.

[18] F. Testard (1986), Polarité, points multiples et géométrie de certain processus gaussiens. Publ. du Laboratoire de Statistique et Probabilités de l'U.P.S. Toulouse, 01-86.

[19] J. B. Walsh (1986), An introduction to stochastic partial differential equations. École d'été de probabilités de Saint-Flour, XIV-1984, pp. 265-439, Lecture Notes in Math., 1180, Springer, Berlin.

[20] D. Wu and Y. Xiao (2006), Fractal properties of random string processes. IMS Lecture NotesMonograph Series-High Dimensional Probability. 51, 128-147.

[21] D. Wu and Y. Xiao (2007), Geometric properties of the images of fractional Brownian sheets. J. Fourier Anal. Appl. 13, 1-37.

[22] Y. Xiao (1999), Hitting probabilities and polar sets for fractional Brownian motion. Stoch. Stoch. Rep. 66, 121-151.

[23] Y. Xiao (2007), Sample path properties of anisotropic Gaussian random fields. In: A Minicourse on Stochastic Partial Differential Equations, (D. Khoshnevisan and F. Rassoul-Agha, editors), Springer, New York. (to appear).

Hermine Biermé: MAP5, Université Paris Descartes, 45 rue des Saints-Pères, 75006 Paris, France

E-mail: hermine.bierme@math-info.univ-paris5.fr

URL: http://www.math-info.univ-paris5.fr/ ${ }^{\sim}$ bierme/

CÉLIne Lacaux: Institut Élie Cartan, Nancy-Université, CNRS, INRIA, Boulevard des Aiguillettes, BP 239, F-54506 Vandoeuvre-Lès-Nancy, France

E-mail: Celine.Lacaux@iecn.u-nancy.fr

URL: http://www.iecn.u-nancy.fr/ lacaux/

Yimin XIAO: Department of Statistics and Probability, A-413 Wells Hall, Michigan State University, East Lansing, MI 48824, U.S.A.

E-mail: xiao@stt.msu.edu

URL: http://www.stt.msu.edu/ xiaoyimi/ 